\title{
$r$-SCAN STATISTICS OF A POISSON PROCESS \\ WITH EVENTS TRANSFORMED BY DUPLICATIONS, DELETIONS, AND DISPLACEMENTS
}

\author{
CHINGFER CHEN ${ }^{*}$ AND \\ SAMUEL KARLIN, ${ }^{* *}$ Stanford University
}

\begin{abstract}
A stochastic model of a dynamic marker array in which markers could disappear, duplicate, and move relative to its original position is constructed to reflect on the nature of long DNA sequences. The sequence changes of deletions, duplications, and displacements follow the stochastic rules: (i) the original distribution of the marker array $\left\{\ldots, X_{-2}, X_{-1}, X_{0}, X_{1}, X_{2}, \ldots\right\}$ is a Poisson process on the real line; (ii) each marker is replicated $l$ times; replication or loss of marker points occur independently; (iii) each replicated point is independently and randomly displaced by an amount $Y$ relative to its original position, with the $Y$ displacements sampled from a continuous density $g(y)$. Limiting distributions for the maximal and minimal statistics of the $r$-scan lengths (collection of distances between $r+1$ successive markers) for the $l$-shift model are derived with the aid of the Chen-Stein method and properties of Poisson processes.
\end{abstract}

Keywords: $r$-scan statistic; Poisson process; Chen-Stein method; Poisson approximation

2000 Mathematics Subject Classification: Primary 60H30

Secondary 60G70

\section{Introduction}

The motivation for the $l$-fold shift model analyzed in this paper stems from the dynamic and heterogenous nature of long DNA sequences. Genomic local and global compositional heterogeneity occurs on many scales. Examples of DNA heterogeneity include isochore compartments (regions dominated by either $\mathrm{G}+\mathrm{C}$ or $\mathrm{A}+\mathrm{T}$ nucleotides as determined by densitygradient centrifugation especially in mammalian species) (Bernardi et al. (1985), (1988)); mobile elements (DNA sequences that move around the genome such as Alu elements in human, Ty sequences in yeast, and IS segments in Escherichia coli (Berg and Howe (1989)); characteristic satellite centrometric tandem repeats (such as the 171-units of human alpha satellite DNA); characteristic telomeric sequences (at the chromosomal termini such as the TGTGGG tandem repeats in humans) (Willard and Waye (1987); Blackburn (1991)); CpG islands (human DNA sequences that occur generally upstream of genes and are abundant with unmethylated CG dinucleotides) (Bird (1986)); repetitive extragenic palindromes (REPs) found in the bacterial genomes of Escherichia coli and Salmonella typhimurium; recombinational hot spots (such as chi elements GCTGGTGG in Escherichia coli) (Krawiec and Riley (1990), Gilson et al. (1991)); almost universal under-representation of the dinucleotide TA; suppression of

Received 11 April 2006; revision received 21 June 2007.

* Postal address: Department of Mathematics, Stanford University, Stanford, CA 94305, USA.

** Email address: Karlin@math.stanford.edu 
the dinucleotide CG in vertebrate species (Josse et al. (1961)); the rarity of the tetranucleotide CTAG in several proteobacterial and archaeal genomes (Burge et al. (1992), Reinert and Schbath (1998)). GNN periodicity in manmalian coding sequences (Ficket (1982)). Thus, genome organization is complex and variegated.

This paper develops a theoretical framework for ascertaining regions of clustering or overdispersion in a marker array (e.g. genes, oligonucletides, transposable elements, and nucleosomes) along a DNA sequence following long-term mutation events such as sequence deletions, duplications, displacements, and rearrangements. To this purpose we consider a Poisson point process model, where each marker is independently replicated a random number of times and the replicas are randomly displaced. The maximal and minimal $r$-scan lengths ( $r$-scans consist of all distances between $r+1$ consecutive points of the marker array) are investigated to identify special inhomogeneous regions. We use multidimensional inhomogeneous Poisson processes in conjunction with the Chen-Stein methodology in characterizing extremal $r$-scans. Moreover, the Kingman mapping theorem (Kingman (1993, Chapter 5)) concerned with transformed Poisson processes in multidimensional spaces is used to achieve essential estimates.

The biological model discussed in this paper describes a stochastic version to these kinds of biological changes and obeys the following rules.

1. The original distribution of the marker array, $\left(\ldots, X_{-2}, X_{-1}, X_{0}, X_{1}, X_{2}, \ldots\right)$, is a point process.

2. Each marker is independently replicated $l$ times. Replications or loss of marker points occur independently.

3. Each replicated point is independently displaced by an amount $Y(-\infty<Y<\infty)$ relative to its original position, with the $Y$ displacements sampled from the density $g(y)$, $-\infty<y<\infty$.

From the altered process, the $r$-scan statistics (see Dembo and Karlin (1992)), are the collection of the interval lengths between all $r+1$ successive marker points. The overdispersion and cluster regions of the marker array correspond to the regions containing the maximal and minimal $r$-scan lengths, respectively. The objective of this paper is to characterize the asymptotic distributions of the maximal and minimal $r$-scan lengths of the shift process. For previous literature and applications of $r$-scan statistics in molecular genetic analysis, see Karlin and Macken (1991), Dembo and Karlin (1992), Karlin and Brendel (1992), Karlin and Cardon (1994), Karlin et al. (1996), and Gerstein (1997). For studies of clustering in other domains with extensive bibliography, see Naus (1979), (1982) and the recent books of Barbour et al. (1992) and Glaz et al. (2001).

We will concentrate on the case in which $A$ (the original ancestor marker array) is distributed as a homogeneous Poisson process of parameter 1 . Let $\Pi_{1}^{(l)}$ denote the shift model constructed from a Poisson(1) process involving an $l$-fold replication and an independent displacement sampled from the density $g$ applied to each replicated point. Thus, the $\Pi_{1}^{(l)}$ array consists of the points

$$
\Pi_{1}^{(l)}=\left\{Z_{i}^{k}=X_{i}+Y_{i}^{k} ; i=0, \pm 1, \pm 2, \ldots, k=1,2, \ldots, l\right\} .
$$

The asymptotic distributions of the extremal $r$-scan lengths descendant from the marker array $\Pi_{1}^{(l)}$ will be deduced from the $l$-dimensional inhomogeneous Poisson process $\Pi_{l}^{*}$ which has the intensity $f_{l}\left(z_{1}, \ldots, z_{l}\right)=\int_{-\infty}^{\infty} g\left(z_{1}-s\right) \cdots g\left(z_{l}-s\right) \mathrm{d} s$. To clarify the ideas and constructions, the case in which $l=2$ will be elaborated. 
The analysis (lemmas and proofs) of the case in which $l>2$ is omitted here, but is available online at http://math.stanford.edu/ karlin/ through the supplemental information link under the publications heading. Consider $l=2$ duplications, producing

$$
\ldots,\left(\begin{array}{l}
X_{-1} \\
X_{-1}
\end{array}\right),\left(\begin{array}{l}
X_{0} \\
X_{0}
\end{array}\right),\left(\begin{array}{l}
X_{1} \\
X_{1}
\end{array}\right),\left(\begin{array}{l}
X_{2} \\
X_{2}
\end{array}\right), \ldots
$$

The displaced array is

$$
\ldots,\left(\begin{array}{l}
Z_{-1}^{1}=X_{-1}+Y_{-1}^{1} \\
Z_{-1}^{2}=X_{-1}+Y_{-1}^{2}
\end{array}\right),\left(\begin{array}{l}
Z_{0}^{1}=X_{0}+Y_{0}^{1} \\
Z_{0}^{2}=X_{0}+Y_{0}^{2}
\end{array}\right),\left(\begin{array}{l}
Z_{1}^{1}=X_{1}+Y_{1}^{1} \\
Z_{1}^{2}=X_{1}+Y_{1}^{2}
\end{array}\right), \ldots,
$$

where all $\{Y\}$ are independent and identically distributed samples from the density $g(y)$. We assume that $\int_{-\infty}^{\infty}|u| g(u) \mathrm{d} u<\infty$ and so $\iint|u-v| g(u) g(v) \mathrm{d} u \mathrm{~d} v<\infty$. It is convenient to introduce the two-dimensional process $\left(X_{i}+Y_{i}^{1}, X_{i}+Y_{i}^{2}\right)$, designated $\Pi_{2}^{*}$, which is an inhomogeneous Poisson process with the intensity rate $f_{2}\left(z_{1}, z_{2}\right)=\int_{-\infty}^{\infty} g\left(z_{1}-s\right) g\left(z_{2}-s\right) \mathrm{d} s$.

Theorem 1. (Asymptotic maximal $r$-scan for the 2-fold shift model.) Let $\Pi_{1}^{(2)}$ be the 2-fold shift process. Assume that the shift length density $g(s)$ satisfies the condition $\int|s| g(s) \mathrm{d} s<\infty$. Then, for any fixed integer $r=2 p+1$, where $p$ is a nonnegative integer, the kth longest $r$-scan length of $\Pi_{1}^{(2)}$ in $(0, t), M_{t, k}$, possesses the asymptotic distribution $(t \rightarrow \infty)$

$$
\lim _{t \rightarrow \infty} \operatorname{Pr}\left\{M_{t, k} \leq \ln t+\left\lfloor\frac{r-1}{2}\right\rfloor \ln \ln t+x\right\}=\sum_{j=0}^{k-1} \mathrm{e}^{-\lambda} \frac{\lambda^{j}}{j !},
$$

where $\lfloor w\rfloor$ is the integer part of $w$ and

$$
\lambda=\frac{\exp \left\{-\left(x+\int_{-\infty}^{\infty} \int_{-\infty}^{\infty}|u-v| g(u) g(v) \mathrm{d} u \mathrm{~d} v\right)\right\}}{\lfloor(r-1) / 2\rfloor !} .
$$

Theorem 2. (Asymptotic minimal $r$-scan for the 2-fold shift model.) Let $\Pi_{1}^{(2)}$ be the 2-fold shift process. Assume that the shift density $g$ is continuous. Then the kth smallest $r$-scan length of $\Pi_{1}^{(2)}$ in $(0, t), m_{t, k}$, possesses the asymptotic distribution

$$
\lim _{t \rightarrow \infty} \operatorname{Pr}\left\{m_{t, k} \geq \sqrt[r]{\frac{x}{t}}\right\}=\sum_{j=0}^{k-1} \mathrm{e}^{-\alpha} \frac{\alpha^{j}}{j !}
$$

with

$$
\alpha=(r+1) x \sum_{j=0}^{\lfloor(r+1) / 2\rfloor} \frac{2^{r+1-2 j}\left[f_{2}(0,0)\right]^{j}}{j !(r+1-2 j) !} .
$$

\subsection{The $l$-fold shift model}

For each marker duplicated $l$ times and each replicate randomly displaced independently as before, we construct a corresponding $l$-dimensional inhomogeneous Poisson process $\Pi_{l}^{*}$ with rate parameter

$$
f_{l}\left(z_{1}, \ldots, z_{l}\right)=\int_{-\infty}^{\infty} g\left(z_{1}-s\right) \cdots g\left(z_{l}-s\right) \mathrm{d} s .
$$


For $1 \leq v \leq l$, let

$$
\begin{aligned}
& S_{v}(z)=\left\{\left(x_{1}, \ldots, x_{l}\right): \text { where the } v \text { th coordinate satisfies } 0 \leq x_{v} \leq z\right\}, \\
& \overline{S_{v}}(z)=\text { the complement of } S_{v}(z), \\
& T_{v}(z)=\cap_{j=1}^{v} S_{j}(z) \cap_{j=v+1}^{l} \overline{S_{j}}(z) .
\end{aligned}
$$

We will establish the existence of the following limits

$$
\begin{aligned}
c_{v} & =\lim _{z \rightarrow \infty} \int \cdots \int_{T_{v}(z)} f_{l}\left(x_{1}, \ldots, x_{l}\right) \mathrm{d} x_{1} \cdots \mathrm{d} x_{l} \quad \text { for } 1 \leq v \leq l-1, \\
c_{l} & =\lim _{z \rightarrow \infty}\left[z-\int \cdots \int_{T_{l}(z)} f_{l}\left(x_{1}, \ldots, x_{l}\right) \mathrm{d} x_{1} \cdots \mathrm{d} x_{l}\right] .
\end{aligned}
$$

Theorem 3. (Asymptotic maximal $r$-scan length of the $l$-fold shift model.) Let $\Pi_{1}^{(l)}$ be the $l$-fold shift process. Suppose that $\int_{-\infty}^{\infty}|s| g(s) \mathrm{d} s<\infty$ and $r=l p+1$, for $p$ a nonnegative integer. Then the kth maximal $r$-scan length from $\Pi_{1}^{(l)}$ in $(0, t), M_{t, k}^{(l)}$, possesses the asymptotic distribution

$$
\lim _{t \rightarrow \infty} \operatorname{Pr}\left\{M_{t, k}^{(l)} \leq \ln t+\left\lfloor\frac{r-1}{l}\right\rfloor \ln \ln t+x\right\}=\sum_{j=0}^{k-1} \mathrm{e}^{-\lambda_{(l)}} \frac{\lambda_{(l)}^{j}}{j !}
$$

with

$$
\lambda_{(l)}=\exp \left\{-\left(x+\sum_{\nu=1}^{l-1}\left(\begin{array}{l}
l \\
v
\end{array}\right) c_{v}-c_{l}\right)\right\} /\left\lfloor\frac{r-1}{l}\right\rfloor !
$$

Theorem 4. (Asymptotic minimal $r$-scan length of the $l$-fold shift model.) Let $\Pi_{1}^{(l)}$ be the $l$-fold shift process. Suppose that the shift density $g$ is continuous. Then the kth minimal $r$-scan length from $\Pi_{1}^{(l)}$ in $(0, t), m_{t, k}^{(l)}$, possesses the asymptotic distribution

$$
\lim _{t \rightarrow \infty} \operatorname{Pr}\left\{m_{t, k}^{(l)} \geq \sqrt[r]{\frac{x}{t}}\right\}=\sum_{j=0}^{k-1} \mathrm{e}^{-\alpha_{(l)}} \frac{\left(\alpha_{(l)}\right)^{j}}{j !}
$$

with

$$
\alpha_{(l)}=(r+1) x \sum_{\substack{i_{1}, i_{2}, \ldots, i_{l} \in \mathbb{Z}^{+} \\
\sum_{v=1}^{l} v i_{v}=r+1}}\left(\prod_{\nu=1}^{l}\left(\left(\begin{array}{l}
l \\
v
\end{array}\right) f_{v}(0, \ldots, 0)^{i_{v}} / i_{v} !\right)\right.
$$

for $f_{d}(0, \ldots, 0)=\int_{-\infty}^{\infty}[g(s)]^{d} \mathrm{~d} s$.

\section{The Chen-Stein method and transformed Poisson processes}

We review first the Chen-Stein method (Chen (1975)), which provides the basic tool to determine the error bound between a sum of (dependent) Bernoulli random variables and its asymptotic Poisson law. In this paper we adopt the formulation of the Chen-Stein method from Arratia et al. (1989). 
Theorem 5. (Chen-Stein method.) Let $\left\{Z_{i}\right\}$ be Bernoulli $\left(p_{i}\right)$ random variables and $W=$ $\sum_{i \in \Omega} Z_{i}$, where $\Omega$ is a finite or countable index set. Let $\mathcal{P}_{\lambda}$ denote the Poisson $(\lambda)$ random variable and let $d(U, V)$ denote the total variation distance between the discrete distributions of $U$ and $V$ :

$$
\begin{aligned}
d(U, V) & \left.=\sup _{\mathcal{A}}(\operatorname{Pr}\{U \in \mathcal{A}\}-\operatorname{Pr}\{V \in \mathcal{A}\}) \quad \text { (where } \mathcal{A} \text { is any measurable set }\right) \\
& =\frac{1}{2} \sum_{k=0}^{\infty}|\operatorname{Pr}\{U=k\}-\operatorname{Pr}\{V=k\}| .
\end{aligned}
$$

Then

where

$$
d\left(W, \mathcal{P}_{\lambda}\right) \leq\left(u_{1}+u_{2}\right) \frac{1-\mathrm{e}^{-\lambda}}{\lambda}+u_{3} \min \left(1, \frac{\sqrt{2}}{\sqrt{\lambda}}\right)
$$

$$
\begin{aligned}
\lambda & =\sum_{i \in \Omega} p_{i}, & u_{1} & =\sum_{i \in \Omega} \sum_{j \in \mathscr{B}_{i}} p_{i} p_{j}, \\
u_{2} & =\sum_{i \in \Omega} \sum_{j \in \mathcal{B}_{i}, j \neq i} \mathrm{E}\left[Z_{i} Z_{j}\right], & u_{3} & =\sum_{i \in \Omega} \mathrm{E}\left[\left|\mathrm{E}\left[Z_{i} \mid\left\{Z_{j}\right\}_{j \notin B_{i}}\right]-p_{i}\right|\right],
\end{aligned}
$$

and $\left\{\mathcal{B}_{i}\right\}$ is an appropriate family of subsets indexed by $\Omega$.

Theorems 6 and 7 are fundamental for inhomogeneous Poisson processes; see, e.g. Kingman (1993, Chapter 5).

Theorem 6. Let $\Pi$ be a Poisson process on a space $S$ with rate measure $\mu$. Suppose that, with each point $X$ of $\Pi$, we associate a random variable $m_{X}$ (the mark of $X$ ) taking values in some metric space $M$. The distribution of $m_{X}$ may depend on $X$ but not on other points of $\Pi$, and $m_{X}$ for different $X$ are independent.

The pair $\left(X, m_{X}\right)$ can be regarded as a random point $X^{*}$ in the product space $S \times M$. Then, the ensemble of points $X^{*}$ generate a Poisson process $\Pi^{*}=\left\{\left(X, m_{X}\right)\right\}_{X \in \Pi}$ on the direct product space $S \times M$ with rate measure $\mu^{*}$ given by

$$
\mu^{*}(C)=\iint_{(x, m) \in C} \mu(\mathrm{d} x) p_{x}(\mathrm{~d} m)
$$

where $p_{x}(\mathrm{~d} m)$ is the conditional distribution of $m$ given $x$.

To adapt Theorem 6 to the 2-fold shift model, we determine $m_{X} \equiv\left(m_{1}, m_{2}\right)=(X+$ $Y_{X}^{1}, X+Y_{X}^{2}$ ), with the displacements $Y_{X}^{1}$ and $Y_{X}^{2}$ arising as independent, real valued, random variables sampled from the density $g(y)$. Then $p_{x}(\mathrm{~d} m)$ applied in (2) is $p_{x}(\mathrm{~d} m)=g\left(m_{1}-\right.$ $x) g\left(m_{2}-x\right) \mathrm{d} m_{1} \mathrm{~d} m_{2}$. The Poisson process $\Pi^{*}$ has the rate measure $\mu^{*}$ on $\mathbb{R} \times \mathbb{R}^{2}$ calculated as

$$
\mu^{*}(C)=\iiint_{\left(x, m_{1}, m_{2}\right) \in C} g\left(m_{1}-x\right) g\left(m_{2}-x\right) \mathrm{d} m_{1} \mathrm{~d} m_{2} \mathrm{~d} x
$$

for any measurable set $C$ on $\mathbb{R}^{3}$.

Theorem 7. (Mapping theorem.) Let $\Pi$ be a Poisson process with a finite mean rate measure $\mu$ on the space $S$, and let $\Lambda: S \rightarrow T$ be a measurable mapping such that the induced measure of $\mu$ transferred to $T$ is atomless. Then $\tilde{\Pi}=\Lambda(\Pi)$ is a Poisson process on $T$ and has rate measure $\tilde{\mu}=\mu * \Lambda^{-1}$. 
In the 2-fold shift model, $\Lambda$ in Theorem 7 is specified as the projection of $\mathbb{R}^{3}$ to $\mathbb{R}^{2}$ such that $\Lambda:\left(x, m_{1}, m_{2}\right) \rightarrow\left(m_{1}, m_{2}\right)$, and therefore the induced process

$$
\Pi_{2}^{*}=\Lambda\left(\left\{X, X+Y_{X}^{1}, X+Y_{X}^{2}\right\}\right)=\left\{\left(X+Y_{X}^{1}, X+Y_{X}^{2}\right)\right\}
$$

is a two-dimensional Poisson process with the rate measure $f_{2}\left(z_{1}, z_{2}\right)=\int_{-\infty}^{\infty} g\left(z_{1}-s\right)$ $\times g\left(z_{2}-s\right) \mathrm{d} s$. We assume that the displacement distribution density $g$ has finite mean. Then $f_{2}$ possesses the following three properties.

1. Symmetry: $f_{2}\left(z_{1}, z_{2}\right)=f_{2}\left(z_{2}, z_{1}\right)$.

2. Invariance under equal translation: $f_{2}\left(z_{1}+a, z_{2}+a\right)=f_{2}\left(z_{1}, z_{2}\right)$ for all real $a$.

3. $f_{2}(z, 0)$ (or $\left.f_{2}(0, z)\right)$ is a continous, symmetric density function of $z$ such that

$$
\int_{-\infty}^{\infty}|z| f_{2}(z, 0) \mathrm{d} z=\int_{-\infty}^{\infty} \int_{-\infty}^{\infty}|u-v| g(u) g(v) \mathrm{d} u \mathrm{~d} v<\infty .
$$

To construct the relevant one-dimensional shift process $\Pi_{1}^{(2)}$, we project the two-dimensional Poisson process $\Pi_{2}^{*}$ separately to its $z_{1}$-axis and to its $z_{2}$-axis and concatenate the two onedimensional point processes yielding the one-dimensional process

$$
\Pi_{1}^{(2)}=\left\{\left\{X+Y_{X}^{1}\right\} \cup\left\{X+Y_{X}^{2}\right\}\right\}_{\left(X+Y_{X}^{1}, X+Y_{X}^{2}\right) \in \Pi_{2}^{*}} .
$$

Theorems 6 and 7 will enable us to study the distributional properties of the two-dimensional Poisson process $\Pi_{2}^{*}$ and, subsequently, to calculate the distribution of the one-dimensional shift process $\Pi_{1}^{(2)}$. For example, consider an interval $(a, b)$, for $a<b$. The two events

$$
\text { \{no one-dimensional } \left.\Pi_{1}^{(2)} \text { point occurs in an interval }(a, b)\right\}
$$

and

$\left\{\right.$ no two-dimensional $\Pi_{2}^{*}$ point occurs in a region of $\{(a, b) \times(-\infty, \infty)\} \cup$

$$
\{(-\infty, \infty) \times(a, b)\}\}
$$

are equivalent. The following notations indicate the regions in $\Pi_{2}^{*}$ associated with the interval $(a, b)$ in $\Pi_{1}^{(2)}$. Let ' $\backslash$ ' denote set subtraction and

$$
\begin{gathered}
A(a, b)=\{(a, b) \times(-\infty, \infty) \cup(-\infty, \infty) \times(a, b)\}, \\
A_{1}(a, b)=\{(a, b) \times(a, b)\}, \quad \text { and } \quad A_{2}(a, b)=A(a, b) \backslash A_{1}(a, b) ;
\end{gathered}
$$

see Figure 1.

Each point of $\Pi_{2}^{*}$ in $A_{1}(a, b)$ corresponds to two points of $\Pi_{1}^{(2)}$ in $(a, b)$ (precluding the points along the diagonal), whereas each point of $\Pi_{2}^{*}$ in $A_{2}(a, b)$ generates a single point of $\Pi_{1}^{(2)}$ in $(a, b)$. Let $V(C)$ equal the integration of $f_{2}\left(z_{1}, z_{2}\right)$ over a set $C$. Since the rate density $f_{2}\left(z_{1}, z_{2}\right)$ of $\Pi_{2}^{*}$ is invariant along the diagonal, it is clear that

$$
V\left(A_{1}(a, b)\right)=V\left(A_{1}(0, b-a)\right) \quad \text { and } \quad V\left(A_{2}(a, b)\right)=V\left(A_{2}(0, b-a)\right) .
$$

Actually, $\Pi_{1}^{(2)}$ is a stationary point process on the line. 


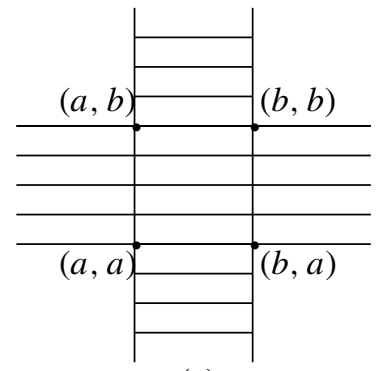

(a)

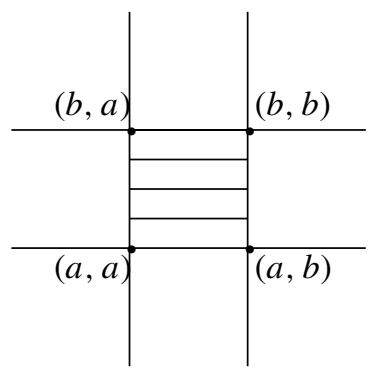

(b)

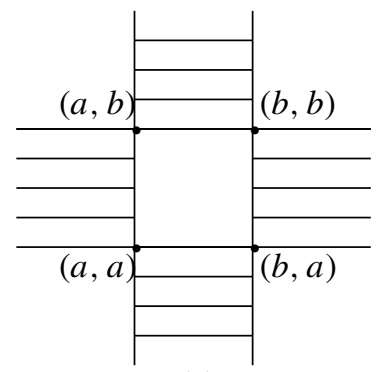

(c)

Figure 1: The stripped regions correspond to (a) $A(a, b)$, (b) $A_{1}(a, b)$, and (c) $A_{2}(a, b)$.

\section{Estimates required for the multiple shift model}

In this section we provide the estimates required to prove Theorems $1-4$. The detailed proof for each estimate is presented in Section 5 of this paper. The analysis of the $r$-scan statistics for the observed marker array $\Pi_{1}^{(2)}$ in (3) is based on the counts of associated Bernoulli variables ((6) and (7), below, over the time horizon $(0, t))$. To study the maximum $r$-scan length, we partition $(0, t)$ with a small spacing $\triangle_{t}=1 / t$ and place a window of width

$$
b_{t}=\ln t+\left\lfloor\frac{r-1}{2}\right\rfloor \ln \ln t+x
$$

at each position $j \triangle_{t}, j=0,1,2, \ldots$ Now, let

$$
Z_{j}^{+}\left(b_{t}\right)= \begin{cases}1 & \text { if there is a single marker of } \Pi_{1}^{(2)} \text { in }\left((j-1) \triangle_{t}, j \triangle_{t}\right), \text { and } \\
\text { less than } r \text { markers in the window of }\left(j \triangle_{t}, j \triangle_{t}+b_{t}\right), \\
0 \quad \begin{array}{l}
\text { otherwise. }
\end{array}\end{cases}
$$

Then $Z_{j}^{+}\left(b_{t}\right)=1$ signifies the existence of an $r$-scan interval of length exceeding $b_{t}$, where its interval begins about $j \triangle_{t}$. We define $n_{t}^{+}\left(b_{t}\right)=\sum_{j=0}^{\left\lfloor\left(t-b_{t}\right) / \Delta_{t}\right\rfloor} Z_{j}^{+}\left(b_{t}\right)$ and prove that $n_{t}^{+}\left(b_{t}\right)$ is a good approximation of $N_{t}^{+}\left(b_{t}\right)$, the count of $r$-scan intervals in $(0, t)$ that exceed $b_{t}$. Theorem 5 can be applied to derive the asymptotic Poisson law for $n_{t}^{+}\left(b_{t}\right)$.

The distribution of the minimum $r$-scan length is studied in a similar manner by partitioning $(0, t)$ with a spacing of $\delta_{t}=1 / t^{2}$ and by putting a window of extent $a_{t}=\sqrt[r]{x / t}$ at each discrete position $j \delta_{t}, j=0,1,2, \ldots$ A Bernoulli random variable is specified at each position $j \delta_{t}$ :

$$
Z_{j}^{-}\left(a_{t}\right)= \begin{cases}1 \quad & \text { if there is a single marker of } \Pi_{1}^{(2)} \text { in }\left((j-1) \delta_{t}, j \delta_{t}\right), \text { and } \\ & \text { at least } r \text { markers in }\left(j \delta_{t},(j-1) \delta_{t}+a_{t}\right), \\ 0 \quad & \text { otherwise. }\end{cases}
$$

When $Z_{j}^{-}\left(a_{t}\right)=1$, there is an $r$-scan interval of length less than $a_{t}$ with its initial marker about $j \delta_{t}$. Let $n_{t}^{-}\left(a_{t}\right)=\sum_{j=0}^{\left\lfloor\left(t-a_{t}\right) / \delta_{t}\right\rfloor} Z_{j}^{-}\left(a_{t}\right)$ and let $N_{t}^{-}\left(a_{t}\right)$ be the count of $r$-scan intervals in $(0, t)$ that do not exceed $a_{t}$. Then $n_{t}^{-}\left(a_{t}\right)$ is a candidate to represent $N_{t}^{-}\left(a_{t}\right)$ such that $n_{t}^{-}\left(a_{t}\right)$ converges to $N_{t}^{-}\left(a_{t}\right)$ in probability as $t \rightarrow \infty$. The asymptotic Poisson law of $n_{t}^{-}\left(a_{t}\right)$ can be ascertained by Theorem 5 .

Before calculating probabilities of events of the process $\Pi_{1}^{(2)}$, we describe concisely the method. Since $\Pi_{2}^{*}$ is a two-dimensional Poisson process, realizations in disjoint areas are 
independent. But points occurring in disjoint intervals of the one-dimensional $\Pi_{1}^{(2)}$, say, $(a, b)$ and $(c, d)$, have some overlap through the rectangle areas $\{(a, b) \times(c, d)\}$ and $\{(c, d) \times(a, b)\}$ of $\Pi_{2}^{*}$, in which each point could project to two one-dimensional points in the intervals $(a, b)$ and $(c, d)$ each. For convenience, we use the notation $A(a, b), A_{1}(a, b)$, and $A_{2}(a, b)$ (see Figure 1 ) to represent regions of $\Pi_{2}^{*}$ relevant to an interval $(a, b)$ in $\Pi_{1}^{(2)}$. We define $B((a, b) \times(c, d))=$ $\{\{(a, b) \times(c, d)\} \cup\{(c, d) \times(a, b)\}\}$, which is the area relative to both intervals $(a, b)$ and $(c, d)$. We use $\|||$ to indicate the count of points in regions of both one-dimensional or twodimensional and higher dimensional; e.g. $\|C\|$ and $\|(a, b)\|$ are the count of points of $\Pi_{2}^{*}$ in area $C$ and the count of points of $\Pi_{1}^{(2)}$ in the interval $(a, b)$, respectively.

In this section we state the upper bounds of errors when applying the Chen-Stein method to the shift processes. The proofs are given in Section 5. Lemmas 1 and 2, below, are necessary to calculate the values of $\left\{\mathrm{E}\left[Z_{j}^{+}\left(b_{t}\right)\right]\right\}_{j \geq 0}$, the expectations of the Bernoulli random variables of maximal $r$-scan lengths.

Lemma 1. Assume that $\int|s| g(s) \mathrm{d} s<\infty$. Then, for each nonnegative integer $k$ and $z \rightarrow \infty$,

$$
\begin{aligned}
& \operatorname{Pr}\left\{\text { There are at most } k \text { points of } \Pi_{1}^{(2)} \text { in }(0, z)\right\} \\
& \quad=\exp \left\{-\left(z+m_{2}\right)\right\} \frac{\left(z-m_{2}\right)^{\lfloor k / 2\rfloor}}{\lfloor k / 2\rfloor !} I_{2}(k)(1+o(1)),
\end{aligned}
$$

where o(1) converges to 0 as $z \rightarrow \infty$, and

$$
m_{2}=\int_{-\infty}^{\infty} \int_{-\infty}^{\infty}|u-v| g(u) g(v) \mathrm{d} u \mathrm{~d} v<\infty ; \quad I_{2}(k)= \begin{cases}1, & k \text { is even }, \\ 1+2 m_{2}, & k \text { is odd } .\end{cases}
$$

Lemma 2. Assume that $\int|s| g(s) \mathrm{d} s<\infty$ and $r=2 p+1, p=0,1,2, \ldots$. Then, for $\triangle_{t}=t^{-1}$ and $b_{t}=\ln t+\lfloor(r-1) / 2\rfloor \ln \ln t+x$,

$$
\begin{aligned}
\mathrm{E}\left[Z_{1}^{+}\left(b_{t}\right)\right]= & \operatorname{Pr}\left\{\text { there is a single point of } \Pi_{1}^{(2)} \text { in }\left(0, \triangle_{t}\right)\right. \text { and at most } \\
& \left.(r-1) \text { points of } \Pi_{1}^{(2)} \text { in }\left(\triangle_{t}, \triangle_{t}+b_{t}\right)\right\} \\
= & \frac{\triangle_{t} \exp \left\{-\left(x+m_{2}\right)\right\}}{t\lfloor(r-1) / 2\rfloor !}(1+o(1)) .
\end{aligned}
$$

The following two lemmas are required for the study of the maximal $r$-scan distribution. Lemma 3 is necessary to evaluate the probability of the event $\left\{n_{t}^{+}\left(b_{t}\right) \neq N_{t}^{+}\left(b_{t}\right)\right\}$. Lemma 4 is required for the calculation of the error bound of $u_{2}$ in (1) with the neighborhood sets $\mathscr{B}_{i\{i>1\}}$ specified as $\mathscr{B}_{i}=\left\{j:|j-i|<\left\lfloor 2 b_{t} / \triangle_{t}\right\rfloor\right\}$ when invoking the Chen-Stein method applied to the Bernoulli sum of $n_{t}^{+}\left(b_{t}\right)=\sum_{j=0}^{\left\lfloor\left(t-b_{t}\right) / \Delta_{t}\right\rfloor} Z_{j}^{+}\left(b_{t}\right)$. Here, and throughout the paper, we consider only the index $i, j$ such that $i \triangle_{t}, j \triangle_{t}$ are within the interval $(0, t)$.

Lemma 3. The following estimates assure convergence in probability of $n_{t}^{+}\left(b_{t}\right)$ to $N_{t}^{+}\left(b_{t}\right)$.

$$
\begin{gathered}
\operatorname{Pr}\left\{\left\|\left(0, \triangle_{t}\right)\right\| \geq 2,\left\|\left(\triangle_{t}, \triangle_{t}+b_{t}\right)\right\| \leq r-1\right\} \leq O\left(\frac{\triangle_{t}^{2}}{t}\right), \\
\operatorname{Pr}\left\{\left\|\left(0, \triangle_{t}\right)\right\|=1,\left\|\left(0, b_{t}\right)\right\| \leq r,\left\|\left(0, \triangle_{t}+b_{t}\right)\right\| \geq r+1\right\} \leq O\left(\frac{\triangle_{t}^{2}}{t}\right) .
\end{gathered}
$$


Lemma 4. The following estimate is necessary for evaluating the parameter $u_{2}$ in (1) when the Chen-Stein method is applied to $n_{t}^{+}\left(b_{t}\right)$ by setting $\triangle_{t}=1 / t$. We obtain

$$
\begin{aligned}
\sum_{j \in B_{i}, j \neq i} \mathrm{E}\left[Z_{i}^{+}\left(b_{t}\right) Z_{j}^{+}\left(b_{t}\right)\right] \leq & 2 \frac{\ln \ln t}{\triangle_{t}} O\left(\frac{\triangle_{t}^{2}}{t \ln t}\right)+2 \frac{b_{t}}{\triangle_{t}} O\left(\frac{\triangle_{t}^{2}(\ln t \ln t)^{\lfloor(r-1) / 2\rfloor}}{t(\ln t)^{2}}\right) \\
& +2 \frac{2 b_{t}}{\triangle_{t}} O\left(\frac{\triangle_{t}^{2}}{t^{2}}\right) .
\end{aligned}
$$

Lemmas 5, 6, and 7 are important for the proof of the asymptotic Poisson law when applying the Chen-Stein method to the Bernoulli sum $\sum_{i=1}^{\left\lfloor\left(t-a_{t}\right) / \delta_{t}\right\rfloor} Z_{i}^{-}\left(a_{t}\right)$. Lemma 5 provides the asymptotic value of $\mathrm{E}\left[Z_{i}^{-}\left(a_{t}\right)\right]$. Lemma 6 gives estimates relevant to the convergence of $n_{t}^{-}\left(a_{t}\right)$ to $N_{t}^{-}\left(a_{t}\right)$ in probability. Lemma 7 provides an upper bound of $u_{2}$ in (1) when applying the Chen-Stein method to $n_{t}^{-}\left(a_{t}\right)$.

Lemma 5. Assume that the shift density $g$ is continuous, then, for $\delta_{t}=t^{-2}, a_{t}=\sqrt[r]{x / t}$, and $t \rightarrow \infty$,

$$
\begin{aligned}
\mathrm{E}\left[Z_{1}^{-}\left(a_{t}\right)\right] & \equiv \operatorname{Pr}\left\{\left\|\left(0, \delta_{t}\right)\right\|=1,\left\|\left(\delta_{t}, a_{t}\right)\right\| \geq r\right\} \\
& =\frac{(r+1) \delta_{t} x}{t}\left(\sum_{j=0}^{\lfloor(r+1) / 2\rfloor} \frac{2^{r+1-2 j}\left(f_{2}(0,0)\right)^{j}}{j !(r+1-2 j) !}\right)(1+o(1)) .
\end{aligned}
$$

Lemma 6. The probability bounds of the events $\left\{\left\|\left(0, \delta_{t}\right)\right\| \geq 2,\left\|\left(\delta_{t}, a_{t}\right)\right\| \geq r-1\right\}$ and $\left\{\left\|\left(0, \delta_{t}\right)\right\|=1,\left\|\left(0, a_{t}\right)\right\|<r+1,\left\|\left(0, a_{t}+\delta_{t}\right)\right\| \geq r+1\right\}$ are respectively

$$
\begin{gathered}
\operatorname{Pr}\left\{\left\|\left(0, \delta_{t}\right)\right\| \geq 2,\left\|\left(\delta_{t}, a_{t}\right)\right\| \geq r-1\right\} \leq O\left(\delta_{t}^{2}\right), \\
\operatorname{Pr}\left\{\left\|\left(0, \delta_{t}\right)\right\|=1,\left\|\left(0, a_{t}\right)\right\|<r+1,\left\|\left(0, a_{t}+\delta_{t}\right)\right\| \geq r+1\right\} \leq O\left(\delta_{t}^{2}\right) .
\end{gathered}
$$

Lemma 7. The following estimate is necessary for evaluating the parameter $u_{2}$ of Theorem 5 when the Chen-Stein method is applied to $n_{t}^{-}\left(a_{t}\right)$. For $2 \leq i \leq\left\lfloor a_{t} / \delta_{t}\right\rfloor+1$,

$$
\mathrm{E}\left[Z_{1}^{-}\left(a_{t}\right) Z_{i}^{-}\left(a_{t}\right)\right] \leq O\left(\delta_{t} \delta_{t} a_{t}^{r}\right) .
$$

With the preparations above, we are ready to validate the limiting theorems of the extremal $r$-scan lengths of the 2-fold shift model.

\section{Theorems for extremal $r$-scan lengths}

\subsection{Extremal distribution of maximum and minimum $\boldsymbol{r}$-scans}

The asymptotic distribution of the $k$ th largest $r$-scan length generated from the 2-fold shift model arises from the sum of Bernoulli random variables associated with the set of discrete times $\left\{j \triangle_{t}\right\}, j=1, \ldots,\left\lfloor\left(t-b_{t}\right) / \triangle_{t}\right\rfloor$, for the choices of $\triangle_{t}=t^{-1}$ and $b_{t}=$ $\ln t+\lfloor(r-1) / 2\rfloor \ln \ln t+x$. Explicitly, for $1 \leq j \leq\left\lfloor\left(t-b_{t}\right) / \triangle_{t}\right\rfloor$, we define the following Bernoulli random variables:

$$
Z_{j}^{+}\left(b_{t}\right)= \begin{cases}1 & \text { if }\left\|\left((j-1) \triangle_{t}, j \triangle_{t}\right)\right\|=1 \text { and }\left\|\left(j \triangle_{t}, j \triangle_{t}+b_{t}\right)\right\|<r \\ 0 & \text { otherwise. }\end{cases}
$$

We claim that the Bernoulli sum $n_{t}^{+}\left(b_{t}\right)=\sum_{j=1}^{\left\lfloor\left(t-b_{t}\right) / \Delta_{t}\right\rfloor} Z_{j}^{+}\left(b_{t}\right)$ is a good approximation to the count $N_{t}^{+}\left(b_{t}\right)$ of the number of $r$-scan intervals in $(0, t)$ based on the points of $\Pi_{1}^{(2)}$ whose 
$r$-scans exceed $b_{t}$. Let $\left\{X_{t, i}\right\}_{i \geq 1}$ be the ordered points of $\Pi_{1}^{(2)}$ in $(0, t)$ and set $X_{t, 0}=0$ for definiteness. Then $\left\{R_{t, i}=X_{t, i-1+r}-X_{t, i-1}\right\}_{i \geq 1}$ are the successive $r$-scan segments along the line. Let $\left\{M_{t, 1}, M_{t, 2}, M_{t, 3}, \ldots\right\}$ be the order statistics for $\left\{R_{t, i}\right\}_{i \geq 1}$ in decreasing order. That is, $M_{t, 1}$ is the largest $r$-scan length of $\Pi_{1}^{(2)}$ in $(0, t)$ and $M_{t, k}$ is the $k$ th largest $r$-scan length. The duality relation guarantees that

$$
\left\{M_{t, k} \leq b_{t}\right\}=\left\{N_{t}^{+}\left(b_{t}\right) \leq k-1\right\} .
$$

If $\int|s| g(s) \mathrm{d} s<\infty$ and $r=2 p+1$, for some nonnegative integer $p$, we will prove that the Bernoulli sum, $n_{t}^{+}\left(b_{t}\right)$, possesses the following two properties.

Property 1. $\lim _{t \rightarrow \infty} \operatorname{Pr}\left\{n_{t}^{+}\left(b_{t}\right) \neq N_{t}^{+}\left(b_{t}\right)\right\}=0$.

Property 2. $n_{t}^{+}\left(b_{t}\right)$ is asymptotically Poisson $(\lambda)$ with

$$
\lambda=\frac{\exp \left\{-\left(x+m_{2}\right)\right\}}{\lfloor(r-1) / 2\rfloor !} \text { for } b_{t} \text { as defined in (5) and } m_{2} \text { as defined in (8). }
$$

With Properties 1 and 2, Theorem 1 can be proved as follows.

Proof of Theorem 1. The duality relation, (15), gives

$$
\begin{aligned}
\lim _{t \rightarrow \infty} \operatorname{Pr}\left\{M_{t, k} \leq b_{t}\right\} & =\lim _{t \rightarrow \infty} \operatorname{Pr}\left\{N_{t}^{+}\left(b_{t}\right) \leq k-1\right\} \\
& =\lim _{t \rightarrow \infty} \operatorname{Pr}\left\{n_{t}^{+}\left(b_{t}\right) \leq k-1\right\} \quad(\text { from Property 1) } \\
& =\sum_{j=0}^{k-1} \mathrm{e}^{-\lambda} \frac{\lambda^{j}}{j !} \quad(\text { from Property } 2),
\end{aligned}
$$

as described in Theorem 1. We now prove Properties 1 and 2.

Proof of Property 1. Under the condition of at least $r$ points of $\Pi_{1}^{(2)}$ in $\left(t-b_{t}, t\right)$, a count $Z_{j}^{+}\left(b_{t}\right)=1$ will not show an $r$-segment extending over the position $t . Z_{j}^{+}\left(b_{t}\right)=1$ signifies a count of an $r$-scan segment in $(0, t)$ with a single point in $\left((j-1) \triangle_{t}, j \triangle_{t}\right)$ and with length exceeding $b_{t}$. Therefore, $\left\{\left\|\left(t-b_{t}, t\right)\right\| \geq r\right\} \subseteq\left\{n_{t}^{+}\left(b_{t}\right) \leq N_{t}^{+}\left(b_{t}\right)\right\}$, which implies that

$$
\left\{n_{t}^{+}\left(b_{t}\right)>N_{t}^{+}\left(b_{t}\right)\right\} \subseteq\left\{\left\|\left(t-b_{t}, t\right)\right\| \leq r-1\right\} .
$$

Thus,

$$
\begin{aligned}
\operatorname{Pr}\left\{n_{t}^{+}\left(b_{t}\right)>N_{t}^{+}\left(b_{t}\right)\right\} & \leq \operatorname{Pr}\left\{\left\|\left(t-b_{t}, t\right)\right\| \leq r-1\right\} \quad \text { (according to (16)) } \\
& =\exp \left\{-\left(b_{t}+m_{2}\right)\right\} \frac{\left(b_{t}-m_{2}\right)^{\lfloor(r-1) / 2\rfloor}}{\lfloor(r-1) / 2\rfloor !}(1+o(1)) \\
& =O\left(\frac{1}{t}\right) \quad(\text { by Lemma } 1 \text { and substituting } \\
& \left.b_{t}=\ln t+\lfloor(r-1) / 2\rfloor \ln \ln t+x\right) .
\end{aligned}
$$

Conversely, the event $\left\{N_{t}^{+}\left(b_{t}\right)>n_{t}^{+}\left(b_{t}\right)\right\}$ occurs only if there exists a $j, 1 \leq j \leq$ $\left\lfloor\left(t-b_{t}\right) / \triangle_{t}\right\rfloor$, such that one of the following two cases hold.

Case 1. $\left\|\left((j-1) \triangle_{t}, j \triangle_{t}\right)\right\| \geq 2$ and $\left\|\left(j \triangle_{t}, j \triangle_{t}+b_{t}\right)\right\| \leq r-1$. 
Case 2. $\left\|\left((j-1) \triangle_{t}, j \triangle_{t}\right)\right\|=1$ and $\left\|\left(j \triangle_{t},(j-1) \triangle_{t}+b_{t}\right)\right\| \leq r-1$ and $\|\left(j \triangle_{t}, j \triangle_{t}+\right.$ $\left.b_{t}\right) \| \geq r$.

For Case $1, Z_{j}^{+}\left(b_{t}\right)=0$ and there is at least one $r$-scan segment starting within the interval $\left((j-1) \triangle_{t}, j \triangle_{t}\right)$ with length exceeding $b_{t}$. Case 2 occurs when $Z_{j}^{+}\left(b_{t}\right)=0$ and there is an $r$-scan interval with its initial marker within the interval $\left((j-1) \triangle_{t}, j \triangle_{t}\right)$ and its last marker within the interval $\left((j-1) \triangle_{t}+b_{t}, j \triangle_{t}+b_{t}\right)$ and its length exceeds $b_{t}$.

As shown in (9) and (10), we have the following estimates:

$\operatorname{Pr}\{$ Case 1 occurs for index $j\}=O\left(\frac{\triangle_{t}^{2}}{t}\right), \quad \operatorname{Pr}\{$ Case 2 occurs for index $j\}=O\left(\frac{\triangle_{t}^{2}}{t}\right)$.

Thus,

$$
\begin{aligned}
\operatorname{Pr}\left\{n_{t}^{+}\left(b_{t}\right)<N_{t}^{+}\left(b_{t}\right)\right\}= & \operatorname{Pr}\{\text { Case } 1 \text { or Case } 2 \text { occurs for some index } j\} \\
\leq & \sum_{j=1}^{\left\lfloor\left(t-b_{t}\right) / \triangle_{t}\right\rfloor} \operatorname{Pr}\{\text { Case } 1 \text { occurs for index } j\} \\
& +\sum_{j=1}^{\left\lfloor\left(t-b_{t}\right) / \triangle_{t}\right\rfloor} \operatorname{Pr}\{\text { Case } 2 \text { occurs for index } j\} \\
= & O\left(\frac{t}{\triangle_{t}}\right) O\left(\frac{\triangle_{t}^{2}}{t}\right)+O\left(\frac{t}{\triangle_{t}}\right) O\left(\frac{\triangle_{t}^{2}}{t}\right) \\
= & O\left(\triangle_{t}\right) \\
= & O\left(\frac{1}{t}\right) \quad(\text { by (9) and (10)). }
\end{aligned}
$$

Therefore, by (17) and (18), we have

$$
\operatorname{Pr}\left\{N_{t}^{+}\left(b_{t}\right) \neq n_{t}^{+}\left(b_{t}\right)\right\}=\operatorname{Pr}\left\{N_{t}^{+}\left(b_{t}\right)<n_{t}^{+}\left(b_{t}\right)\right\}+\operatorname{Pr}\left\{N_{t}^{+}\left(b_{t}\right)>n_{t}^{+}\left(b_{t}\right)\right\}=O\left(\frac{1}{t}\right) .
$$

This completes the proof of Property 1.

Proof of Property 2. Assuming that $\int|s| g(s) \mathrm{d} s<\infty$ and $r=2 p+1$, for some nonnegative integer $p$, we apply the Chen-Stein method to verify the asymptotic Poisson law of $n_{t}^{+}\left(b_{t}\right)=$ $\sum_{j=1}^{\left\lfloor\left(t-b_{t}\right) / \Delta_{t}\right\rfloor} Z_{j}^{+}\left(b_{t}\right)$. Following the result of Lemma 2 and the stationarity of $\Pi_{1}^{(2)}$, we have

$$
\mathrm{E}\left[Z_{j}^{+}\left(b_{t}\right)\right]=\mathrm{E}\left[Z_{1}^{+}\left(b_{t}\right)\right]=\frac{\triangle_{t} \exp \left\{-\left(x+m_{2}\right)\right\}}{\lfloor(r-1) / 2\rfloor ! t}(1+o(1)) .
$$

Therefore,

$$
\lambda_{t} \equiv \mathrm{E}\left[n_{t}^{+}\left(b_{t}\right)\right]=\left\lfloor\frac{t-b_{t}}{\triangle_{t}}\right\rfloor \mathrm{E}\left[Z_{1}^{+}\left(b_{t}\right)\right]=\lambda(1+o(1)) \quad \text { for } \lambda=\frac{\exp \left\{-\left(x+m_{2}\right)\right\}}{\lfloor(r-1) / 2\rfloor !} .
$$

To demonstrate the Poisson approximation, we construct an index neighborhood subset $\mathscr{B}_{j}$ for each $j, \mathscr{B}_{j}=\left\{i:|i-j| \leq\left\lfloor 2 b_{t} / \triangle_{t}\right\rfloor\right\}$. According to the Chen-Stein protocol 
(see Barbour et al. (1992)), the upper bound for the total variational distance between $n_{t}^{+}\left(b_{t}\right)$ and a Poisson random variable $\operatorname{Po}(\lambda)$ is

$$
\begin{aligned}
d\left(n_{t}^{+}\left(b_{t}\right), \operatorname{Po}(\lambda)\right) & \leq d\left(n_{t}^{+}\left(b_{t}\right), \operatorname{Po}\left(\lambda_{t}\right)\right)+d\left(\operatorname{Po}\left(\lambda_{t}\right), \operatorname{Po}(\lambda)\right) \\
& \leq\left(u_{1}+u_{2}\right)\left(\frac{1-\mathrm{e}^{-\lambda_{t}}}{\lambda_{t}}\right)+u_{3} \min \left(1, \sqrt{\lambda_{t}}\right)+\left|\lambda-\lambda_{t}\right|,
\end{aligned}
$$

where

$$
\begin{aligned}
& u_{1}=\sum_{j=1}^{\left\lfloor\left(t-b_{t}\right) / \Delta_{t}\right\rfloor} \sum_{i \in B_{j}} \mathrm{E}\left[Z_{j}^{+}\left(b_{t}\right)\right] \mathrm{E}\left[Z_{i}^{+}\left(b_{t}\right)\right], \quad u_{2}=\sum_{j=1}^{\left\lfloor\left(t-b_{t}\right) / \Delta_{t}\right\rfloor} \sum_{i \in \mathcal{B}_{j}, i \neq j} \mathrm{E}\left[Z_{j}^{+}\left(b_{t}\right) Z_{i}^{+}\left(b_{t}\right)\right], \\
& \text { and } u_{3}=2 \sum_{j=1}^{\left\lfloor\left(t-b_{t}\right) / \Delta_{t}\right\rfloor} \mathrm{E}\left[Z_{j}^{+}\left(b_{t}\right)\right] d\left(\left(\sum_{i \notin \mathcal{B}_{j}} Z_{i}^{+}\left(b_{t}\right) \mid Z_{j}^{+}\left(b_{t}\right)=1\right), \sum_{i \notin \mathcal{B}_{j}} Z_{i}^{+}\left(b_{t}\right)\right) .
\end{aligned}
$$

Estimates of $u_{1}, u_{2}$, and $u_{3}$ are assessed by evaluation of corresponding areas of the twodimensional process $\Pi_{2}^{*}$. We use Lemma 2 and Lemma 4 to provide the following estimates:

$$
\begin{aligned}
& u_{1}=\sum_{j=1}^{\left\lfloor\left(t-b_{t}\right) / \Delta_{t}\right\rfloor} \sum_{i \in B_{j}} \mathrm{E}\left[Z_{j}^{+}\left(b_{t}\right)\right] \mathrm{E}\left[Z_{i}^{+}\left(b_{t}\right)\right] \\
& \leq 2\left(\frac{t-b_{t}}{\triangle_{t}}\right)\left(\frac{2 b_{t}}{\triangle_{t}}\right)\left(\mathrm{E}\left[Z_{1}^{+}\left(b_{t}\right)\right]\right)^{2} \quad \text { (by stationarity) } \\
& \leq 4 O\left(\frac{t b_{t}}{\triangle_{t}^{2}}\right)\left(\frac{\triangle_{t}}{t} \lambda\right)^{2} \quad\left(\text { by (19), (20), and } \lambda=\frac{\exp \left\{-\left(x+m_{2}\right)\right\}}{\lfloor(r-1) / 2\rfloor !}\right) \\
& \leq 4 O\left(\frac{\ln t}{t}\right) \lambda^{2} \\
& u_{2}=\sum_{j=1}^{\left\lfloor\left(t-b_{t}\right) / \triangle_{t}\right\rfloor} \sum_{i \in B_{j}, i \neq j} \mathrm{E}\left[Z_{j}^{+}\left(b_{t}\right) Z_{i}^{+}\left(b_{t}\right)\right] \\
& \leq 2 \frac{t}{\triangle_{t}} \sum_{i=2}^{\left\lfloor\left(2 b_{t}\right) / \Delta_{t}\right\rfloor+1} \mathrm{E}\left[Z_{1}^{+}\left(b_{t}\right) Z_{i}^{+}\left(b_{t}\right)\right] \quad \text { (by stationarity) } \\
& =2 \frac{t}{\triangle_{t}}\left\{\sum_{i=2}^{\left\lfloor\ln \ln t / \Delta_{t}\right\rfloor+1} \mathrm{E}\left[Z_{1}^{+}\left(b_{t}\right) Z_{i}^{+}\left(b_{t}\right)\right]+\sum_{\left\lfloor\ln \ln t / \Delta_{t}\right\rfloor+2}^{\left\lfloor b_{t} / \Delta_{t}\right\rfloor+1} \mathrm{E}\left[Z_{1}^{+}\left(b_{t}\right) Z_{i}^{+}\left(b_{t}\right)\right]\right. \\
& \left.+\sum_{\left\lfloor b_{t} / \triangle_{t}\right\rfloor+2}^{\left\lfloor 2 b_{t} / \triangle_{t}\right\rfloor+1} \mathrm{E}\left[Z_{1}^{+}\left(b_{t}\right) Z_{i}^{+}\left(b_{t}\right)\right]\right\} \\
& \leq 2 \frac{t}{\triangle_{t}}\left(\frac{\ln \ln t}{\triangle_{t}} O\left(\frac{\triangle_{t}^{2}}{t \ln t}\right)+\frac{b_{t}}{\triangle_{t}} O\left(\frac{\triangle_{t}^{2}(\ln \ln t)^{(r-1) / 2}}{t(\ln t)^{2}}\right)+\frac{2 b_{t}}{\triangle_{t}} O\left(\frac{\triangle_{t}^{2}}{t^{2}}\right)\right) \\
& =O\left(\frac{\ln \ln t}{\ln t}+\frac{(\ln \ln t)^{(r-1) / 2}}{\ln t}\right) \text {. }
\end{aligned}
$$


To study the convergence property of $u_{3}$, for $j \leq\left\lfloor 2 b_{t} / \triangle_{t}\right\rfloor$, let $E_{j, 1}$ and $E_{j, 2}$ be sets in $\mathbb{R}^{2}$ determined as

$$
\begin{aligned}
& E_{j, 1}=\left\{\left((j-1) \triangle_{t}, j \triangle_{t}+b_{t}\right) \times\left(\left(j-1+\left\lfloor\frac{2 b_{t}}{\triangle_{t}}\right\rfloor\right) \triangle_{t}, \infty\right)\right\} \\
& E_{j, 2}=\left\{\left(\left(j-1+\left\lfloor\frac{2 b_{t}}{\triangle_{t}}\right\rfloor\right) \triangle_{t}, \infty\right) \times\left((j-1) \triangle_{t}, j \triangle_{t}+b_{t}\right)\right\} .
\end{aligned}
$$

And, for $\left\lfloor 2 b_{t} / \triangle_{t}\right\rfloor<j \leq\left\lfloor\left(t-b_{t}\right) / \triangle_{t}\right\rfloor$, let $E_{j, 1}$ and $E_{j, 2}$ be determined as above and let $E_{j, 3}$ and $E_{j, 4}$ be determined as

$$
\begin{aligned}
& E_{j, 3}=\left\{\left((j-1) \triangle_{t}, j \triangle_{t}+b_{t}\right) \times\left(0,\left(j-1-\left\lfloor\frac{2 b_{t}}{\triangle_{t}}\right\rfloor\right) \triangle_{t}\right)\right\}, \\
& E_{j, 4}=\left\{\left(0,\left(j-1-\left\lfloor\frac{2 b_{t}}{\triangle_{t}}\right\rfloor\right) \triangle_{t}\right) \times\left((j-1) \triangle_{t}, j \triangle_{t}+b_{t}\right)\right\} .
\end{aligned}
$$

Then the random variables $Z_{j}^{+}$and $\left\{Z_{i}^{+}\right\}_{\left\{i \notin \mathcal{B}_{j}\right\}}$ interact through these regions. Conditioning on the event that there is no point in $E_{j, 1} \cup E_{j, 2} \cup E_{j, 3} \cup E_{j, 4}$ (that is $\left\|E_{j, 1} \cup E_{j, 2} \cup E_{j, 3} \cup E_{j, 4}\right\|=0$ ), the random variables $Z_{j}^{+}$and $\left\{Z_{i}^{+}\right\}_{\left\{i \notin \mathscr{B}_{j}\right\}}$ are independent. A direct calculation shows that

$$
\begin{aligned}
V\left(E_{j, 2}\right)=V\left(E_{j, 1}\right) & =\iint_{(x, y) \in E_{j, 1}} f_{2}(x, y) \mathrm{d} x \mathrm{~d} y \\
& =\int_{(j-1) \triangle_{t}}^{j \triangle_{t}+b_{t}} \int_{\left(j-1+\left\lfloor 2 b_{t} / \triangle_{t}\right\rfloor\right) \triangle_{t}}^{\infty} f_{2}(x, y) \mathrm{d} y \mathrm{~d} x \\
& =\int_{(j-1) \triangle_{t}}^{j \triangle_{t}+b_{t}} \int_{\left(j-1+\left\lfloor 2 b_{t} / \triangle_{t}\right\rfloor\right) \triangle_{t}}^{\infty} f_{2}(0, y-x) \mathrm{d} y \mathrm{~d} x
\end{aligned}
$$

and since $f_{2}$ is nonnegative and $(y-x, \infty) \subset\left(b_{t}-2 \triangle_{t}, \infty\right)$

$$
\begin{aligned}
& <\int_{(j-1) \triangle_{t}}^{j \triangle_{t}+b_{t}} \int_{b_{t}-2 \triangle_{t}}^{\infty} f_{2}(0, v) \mathrm{d} v \mathrm{~d} x \\
& =\int_{b_{t}-2 \triangle_{t}}^{\infty}\left(\triangle_{t}+b_{t}\right) f_{2}(0, v) \mathrm{d} v \\
& \leq \int_{b_{t}-2 \triangle_{t}}^{\infty}\left(3 \triangle_{t}+v\right) f_{2}(0, v) \mathrm{d} v \quad\left(\text { since } v \geq b_{t}-2 \triangle_{t}\right. \text { and } \\
& \left.3 \triangle_{t}+v \geq \triangle_{t}+b_{t}\right) .
\end{aligned}
$$

Similarly,

$$
V\left(E_{j, 3}\right)=V\left(E_{j, 4}\right) \leq \int_{2 b_{t}}^{\infty}\left(\triangle_{t}+b_{t}\right) f_{2}(0, v) \mathrm{d} v \leq \int_{2 b_{t}}^{\infty} v f_{2}(0, v) \mathrm{d} v .
$$

Since $\int_{0}^{\infty} v f_{2}(0, v) \mathrm{d} v$ is finite, $V\left(E_{j, 1}\right), V\left(E_{j, 2}\right), V\left(E_{j, 3}\right)$, and $V\left(E_{j, 4}\right)$ all converge to 0 as $t \rightarrow \infty$. 
Under the event $\mathscr{E}:\left\{\Pi_{2}^{*} \cap\left\{E_{j, 1} \cup E_{j, 2} \cup E_{j, 3} \cup E_{j, 4}\right\}=\varnothing\right\}, Z_{j}^{+}\left(b_{t}\right)$ and $\sum_{i \notin \mathcal{B}_{j}} Z_{i}^{+}\left(b_{t}\right)$ are independent, i.e. $\left(\sum_{i \notin \mathcal{B}_{j}} Z_{i}^{+}\left(b_{t}\right) \mid \mathcal{E}\right)$ and $\left(Z_{j}^{+}\left(b_{t}\right) \mid \mathcal{E}\right)$ are independent. Therefore,

$$
\begin{aligned}
& d\left(\left(\sum_{i \notin \mathcal{B}_{j}} Z_{i}^{+}\left(b_{t}\right)\left|Z_{j}^{+}\left(b_{t}\right)=1\right| \mathcal{E}\right),\left(\sum_{i \notin \mathcal{B}_{j}} Z_{i}^{+}\left(b_{t}\right) \mid \mathcal{E}\right)\right) \\
& \quad=d\left(\left(\left(\sum_{i \notin \mathcal{B}_{j}} Z_{i}^{+}\left(b_{t}\right) \mid \mathcal{E}\right) \mid\left(Z_{j}^{+}\left(b_{t}\right)=1 \mid \mathcal{E}\right)\right),\left(\sum_{i \notin \mathcal{B}_{j}} Z_{i}^{+}\left(b_{t}\right) \mid \mathcal{E}\right)\right) \\
& \quad=0 .
\end{aligned}
$$

Let $\mathcal{E}^{\mathrm{c}}$ denote the complementary event of $\mathcal{E}$. We then have

$$
\begin{aligned}
& d\left(\left(\sum_{i \notin B_{j}} Z_{i}^{+}\left(b_{t}\right) \mid Z_{j}^{+}\left(b_{t}\right)=1\right), \sum_{i \notin B_{j}} Z_{i}^{+}\left(b_{t}\right)\right) \\
& \leq d\left(\left(\sum_{i \notin \mathcal{B}_{j}} Z_{i}^{+}\left(b_{t}\right)\left|Z_{j}^{+}\left(b_{t}\right)=1\right| \mathcal{E}\right),\left(\sum_{i \notin \mathcal{B}_{j}} Z_{i}^{+}\left(b_{t}\right) \mid \mathcal{E}\right)\right) \operatorname{Pr}\{\mathcal{E}\} \\
& \quad+d\left(\left(\sum_{i \notin \mathcal{B}_{j}} Z_{i}^{+}\left(b_{t}\right)\left|Z_{j}^{+}\left(b_{t}\right)=1\right| \mathcal{E}^{\mathrm{c}}\right),\left(\sum_{i \notin \mathcal{B}_{j}} Z_{i}^{+}\left(b_{t}\right) \mid \mathcal{E}^{\mathrm{c}}\right)\right) \operatorname{Pr}\left\{\mathcal{E}^{\mathrm{c}}\right\} \\
& \quad \leq \operatorname{Pr}\left\{\mathcal{E}^{\mathrm{c}}\right\} \\
& =\operatorname{Pr}\left\{\Pi_{2}^{*} \cap\left\{E_{1} \cup E_{2} \cup E_{3} \cup E_{4}\right\} \neq \varnothing\right\} \\
& =1-\exp \left(-V\left(E_{j, 1} \cup E_{j, 2} \cup E_{j, 3} \cup E_{j, 4}\right)\right) \\
& \quad \longrightarrow 0 .
\end{aligned}
$$

This proves the convergence of $u_{3}$ to 0 . Therefore, the Poisson approximation of $n_{t}^{+}\left(b_{t}\right)$ is proved. It has been proved in (20) that $\lim _{t \rightarrow \infty} \lambda_{t}=\lambda$. Therefore, we have the Poisson distribution with parameter $\lambda$ for $n_{t}^{+}\left(b_{t}\right)$ with $b_{t}=\ln t+\lfloor(r-1) / 2\rfloor \ln \ln t+x$. This completes the proof.

\subsection{Asymtotic minimum $r$-scan distribution}

For the asymptotic distribution of the minimum $r$-scan length of $\Pi_{1}^{(2)}$, we set the partition length $\delta_{t}=t^{-2}$ and $a_{t}=\sqrt[r]{x / t}$. The associated Bernoulli random variables, for $1 \leq j \leq$ $\left\lfloor\left(t-a_{t}\right) / \delta_{t}\right\rfloor$, are

$$
Z_{j}^{-}\left(a_{t}\right)= \begin{cases}1 & \text { if }\left\|\left((j-1) \delta_{t}, j \delta_{t}\right)\right\|=1 \text { and }\left\|\left(j \delta_{t},(j-1) \delta_{t}+a_{t}\right)\right\| \geq r \\ 0 & \text { otherwise. }\end{cases}
$$

We form the sum $n_{t}^{-}\left(a_{t}\right)=\sum_{j=1}^{\left\lfloor\left(t-a_{t}\right) / \delta_{t}\right\rfloor} Z_{j}^{-}\left(a_{t}\right)$, and define $N_{t}^{-}\left(a_{t}\right)$ as the count of $r$-scan intervals which do not exceed $a_{t}$ in the interval $(0, t)$ generated from the points of $\Pi_{1}^{(2)}$.

If the shift density $g$ is continuous, the asymptotic distribution of the $k$ th smallest $r$-scan length of the 2-fold shift model will be based on the following two properties.

Property 3. $\lim _{t \rightarrow \infty} \operatorname{Pr}\left\{N_{t}^{-}\left(a_{t}\right) \neq n_{t}^{-}\left(a_{t}\right)\right\}=0$. 
Property 4. $n_{t}^{-}\left(a_{t}\right)$ is distributed asymptotically Poisson with parameter

$$
\alpha=(r+1) x \sum_{j=0}^{\lfloor(r+1) / 2\rfloor} \frac{2^{r+1-2 j}\left[f_{2}(0,0)\right]^{j}}{j !(r+1-2 j) !} .
$$

From Properties 3 and 4, Theorem 2 can be proved as follows.

Proof of Theorem 2. The duality relation shows that $\operatorname{Pr}\left\{m_{t, k} \geq a_{t}\right\}=\operatorname{Pr}\left\{N_{t}^{-}\left(a_{t}\right) \leq k-1\right\}$. Also,

$$
\begin{aligned}
\lim _{t \rightarrow \infty} \operatorname{Pr}\left\{N_{t}^{-}\left(a_{t}\right) \leq k-1\right\} & \left.=\lim _{t \rightarrow \infty} \operatorname{Pr}\left\{n_{t}^{-}\left(a_{t}\right) \leq k-1\right\} \quad \text { (from Property } 3\right) \\
& =\sum_{j=0}^{k-1} \mathrm{e}^{-\alpha} \frac{\alpha^{j}}{j !} \quad \text { (from Property 4). }
\end{aligned}
$$

Therefore,

$$
\lim _{t \rightarrow \infty} \operatorname{Pr}\left\{m_{t, k} \geq a_{t}\right\}=\sum_{j=0}^{k-1} \mathrm{e}^{-\alpha} \frac{\alpha^{j}}{j !},
$$

as described in Theorem 2 .

Proof of Property 3. The event of $Z_{j}^{-}\left(a_{t}\right)=1$ indicates the existence of an $r$-scan interval with its initial marker in $\left((j-1) \delta_{t}, j \delta_{t}\right)$ and its length less than $a_{t}$. Therefore, we have $N_{t}^{-}\left(a_{t}\right) \geq n_{t}^{-}\left(a_{t}\right)$. Thus, $\operatorname{Pr}\left\{N_{t}^{-}\left(a_{t}\right) \neq n_{t}^{-}\left(a_{t}\right)\right\}=\operatorname{Pr}\left\{N_{t}^{-}\left(a_{t}\right)>n_{t}^{-}\left(a_{t}\right)\right\}$. The event of $\left\{N_{t}^{-}\left(a_{t}\right)>n_{t}^{-}\left(a_{t}\right)\right\}$ will occur only if there exists a $j, 1 \leq j \leq\left\lfloor\left(t-a_{t}\right) / \delta_{t}\right\rfloor$, such that one of the following two events occur.

Case 3. $\left\|\left((j-1) \delta_{t}, j \delta_{t}\right)\right\| \geq 2$ and $\left\|\left(j \delta_{t}, j \delta_{t}+a_{t}\right)\right\| \geq r-1$.

Case 4. $\left\|\left((j-1) \delta_{t}, j \delta_{t}\right)\right\|=1$ and $\left\|\left(j \delta_{t},(j-1) \delta_{t}+a_{t}\right)\right\|<r$ and $\left\|\left(j \delta_{t}, j \delta_{t}+a_{t}\right)\right\| \geq r$.

Case 3 occurs when $Z_{j}^{-}\left(a_{t}\right)=0$ and there is an $r$-scan segment which qualifies for length less than $a_{t}$ and with its first two markers very close together and occurring in $\left((j-1) \delta_{t}, j \delta_{t}\right)$. Here $Z_{j}^{-}\left(a_{t}\right)=1$ indicates the existence of an $r$-scan interval with the first point in the subinterval $\left.\left((j-1) \delta_{t}, j \delta_{t}\right)\right)$ and the $r$-scan length less than $a_{t}$. The probability difference is bounded by

$$
\operatorname{Pr}\left\{Z_{j}^{-}\left(a_{t}\right)=0,\left\|\left((j-1) \delta_{t}, j \delta_{t}\right)\right\|=1,\left\|\left(j \delta_{t}, j \delta_{t}+a_{t}\right)\right\| \geq r\right\}=\operatorname{Pr}\{\text { Case } 4\} .
$$

As shown in (12) and (13), the estimates of $\operatorname{Pr}\{$ Case 3 occurs for index $j\} \leq O\left(\delta_{t}^{2}\right)$ and $\operatorname{Pr}\{$ Case 4 occurs for index $j\} \leq O\left(\delta_{t}^{2}\right)$ prevail. Therefore,

$$
\begin{aligned}
\operatorname{Pr}\left\{N_{t}^{-}\right. & \left.\left(a_{t}\right) \neq n_{t}^{-}\left(a_{t}\right)\right\} \\
= & \operatorname{Pr}\left\{N_{t}^{-}\left(a_{t}\right)>n_{t}^{-}\left(a_{t}\right)\right\} \\
\leq & \operatorname{Pr}\left\{\text { Case } 3 \text { occurs for some index } j, 1 \leq j \leq\left\lfloor\frac{t-a_{t}}{\delta_{t}}\right\rfloor\right\} \\
& \quad+\operatorname{Pr}\left\{\text { Case } 4 \text { occurs for some index } j, 1 \leq j \leq\left\lfloor\frac{t-a_{t}}{\delta_{t}}\right\rfloor\right\} \\
\leq & \sum_{j=1}^{\left\lfloor\left(t-a_{t}\right) / \delta_{t}\right\rfloor} \operatorname{Pr}\{\text { Case } 3 \text { occurs for index } j\}+\sum_{j=1}^{\left\lfloor\left(t-a_{t}\right) / \delta_{t}\right\rfloor} \operatorname{Pr}\{\text { Case } 4 \text { occurs for index } j\}
\end{aligned}
$$




$$
\begin{aligned}
& \leq O\left(\frac{t}{\delta_{t}}\right) O\left(\delta_{t}^{2}\right)+O\left(\frac{t}{\delta_{t}}\right) O\left(\delta_{t}^{2}\right) \\
& =O\left(t^{-1}\right) .
\end{aligned}
$$

This completes the proof.

Proof of Property 4. We will apply the Chen-Stein method to the Bernoulli sum $n_{t}^{-}\left(a_{t}\right)=$ $\sum_{j=1}^{\left\lfloor\left(t-a_{t}\right) / \delta_{t}\right\rfloor} Z_{j}^{-}\left(a_{t}\right)$ for $a_{t}=\sqrt[r]{x / t}$ and $\delta_{t}=t^{-2}$. According to Lemma 5 and the stationary property of $\Pi_{1}^{(2)}$, we have

$$
\mathrm{E}\left[Z_{j}^{-}\left(a_{t}\right)\right]=\mathrm{E}\left[Z_{1}^{-}\left(a_{t}\right)\right]=(r+1) \delta_{t} \frac{x}{t}\left(\sum_{j=0}^{\lfloor(r+1) / 2\rfloor} \frac{2^{r+1-2 j}\left[f_{2}(0,0)\right]^{j}}{j !(r+1-2 j) !}\right)(1+o(1)) .
$$

Therefore,

$$
\begin{aligned}
\alpha_{t}:=\mathrm{E}\left[n_{t}^{-}\left(a_{t}\right)\right] & =\mathrm{E}\left[\sum_{j=1}^{\left\lfloor\left(t-a_{t}\right) / \delta_{t}\right\rfloor} Z_{j}^{-}\left(a_{t}\right)\right] \\
& =\sum_{j=1}^{\left\lfloor(t-\sqrt[r]{x / t}) / \delta_{t}\right\rfloor} \mathrm{E}\left[Z_{j}^{-}\left(a_{t}\right)\right] \\
& =\left(\frac{t}{\delta_{t}}\right) \mathrm{E}\left[Z_{1}^{-}\left(a_{t}\right)\right](1+o(1)) \\
& =\alpha(1+o(1))
\end{aligned}
$$

for

$$
\alpha=(r+1) x \sum_{j=0}^{\lfloor(r+1) / 2\rfloor} \frac{2^{r+1-2 j}\left[f_{2}(0,0)\right]^{j}}{(r+1-2 j) ! j !} .
$$

To validate the Poisson approximation of $n_{t}^{-}\left(a_{t}\right)$, we construct the set of neighborhoods $\left\{\mathscr{D}_{j}\right\}$ for each $j$ as $\mathscr{D}_{j}=\left\{i:|i-j| \leq\left\lfloor a_{t} / \delta_{t}\right\rfloor\right\}$. Then, according to the Chen-Stein protocol (see Theorem 5), the upper bound for the total variational distance between the Bernoulli sum $n_{t}^{-}\left(a_{t}\right)$ and the Poisson $\operatorname{Po}(\alpha)$ random variable is as follows:

$$
\begin{aligned}
d\left(n_{t}^{-}\left(a_{t}\right), \operatorname{Po}(\alpha)\right) & \leq d\left(n_{t}^{-}\left(a_{t}\right), \operatorname{Po}\left(\alpha_{t}\right)\right)+d\left(\operatorname{Po}\left(\alpha_{t}\right), \operatorname{Po}(\alpha)\right) \\
& \leq\left(v_{1}+v_{2}\right)\left(\frac{1-\mathrm{e}^{-\alpha_{t}}}{\alpha_{t}}\right)+v_{3} \min \left(1, \alpha_{t}^{-1 / 2}\right)+\left|\alpha-\alpha_{t}\right|,
\end{aligned}
$$

where

$$
\begin{aligned}
& v_{1}=\sum_{j=1}^{\left\lfloor\left(t-a_{t}\right) / \delta_{t}\right\rfloor} \sum_{i \in D_{j}} \mathrm{E}\left[Z_{j}^{-}\left(a_{t}\right)\right] \mathrm{E}\left[Z_{i}^{-}\left(a_{t}\right)\right], \\
& v_{2}=\sum_{j=1}^{\left\lfloor\left(t-a_{t}\right) / \delta_{t}\right\rfloor} \sum_{i \in \mathcal{D}_{j}, i \neq j} \mathrm{E}\left[Z_{j}^{-}\left(a_{t}\right) Z_{i}^{-}\left(a_{t}\right)\right], \quad \text { and } \\
& v_{3}=2 \sum_{j=1}^{\left\lfloor\left(t-a_{t}\right) / \delta_{t}\right\rfloor} \mathrm{E}\left[Z_{j}^{-}\left(a_{t}\right)\right] d\left(\left(\sum_{i \notin \mathcal{D}_{j}} Z_{i}^{-}\left(a_{t}\right) \mid Z_{j}^{-}\left(a_{t}\right)=1\right), \sum_{i \notin \mathcal{D}_{j}} Z_{i}^{-}\left(a_{t}\right)\right) .
\end{aligned}
$$


Detailed estimates of $v_{1}, v_{2}$, and $v_{3}$ are calculated by evaluating appropriate events of the two-dimensional Poisson process $\Pi_{2}^{*}$. We use Lemmas 5, 6, and 7 to provide the following estimates:

$$
\begin{aligned}
& v_{1}=\sum_{j=1}^{\left\lfloor\left(t-a_{t}\right) / \delta_{t}\right\rfloor} \sum_{i \in D_{j}} \mathrm{E}\left[Z_{j}^{-}\left(a_{t}\right)\right] \mathrm{E}\left[Z_{i}^{-}\left(a_{t}\right)\right] \\
& \leq 2\left(\frac{t}{\delta_{t}}\right)\left(\frac{a_{t}}{\delta_{t}}\right)\left(\mathrm{E}\left[Z_{1}^{-}\left(a_{t}\right)\right]\right)^{2} \quad\left(\text { by the stationary property of } \Pi_{1}^{(2)}\right) \\
& =2\left(\frac{t}{\delta_{t}}\right)\left(\frac{a_{t}}{\delta_{t}}\right)\left(\alpha \frac{\delta_{t}}{t}\right)^{2}(1+o(1)) \quad \text { (by Lemma 5, with } \alpha \text { as defined in (21)) } \\
& =O\left(t^{-(r+1) / r}\right) \text {, } \\
& v_{2}=\sum_{j=1}^{\left\lfloor(t-\sqrt[r]{x / t}) / \delta_{t}\right\rfloor} \sum_{i \in D_{j}, i \neq j} \mathrm{E}\left[Z_{j}^{-}\left(a_{t}\right) Z_{i}^{-}\left(a_{t}\right)\right] \\
& \leq 2\left(\frac{t}{\delta_{t}}\right)\left(\frac{a_{t}}{\delta_{t}}\right) O\left(\delta_{t}^{2} t^{-1}\right) \quad \text { (by (14) in Lemma 7) } \\
& =O\left(t^{-1 / r}\right) \text {. }
\end{aligned}
$$

To verify the convergence of $v_{3}$ to 0 , let $\tilde{E}_{j, 1}, \tilde{E}_{j, 2}, \tilde{E}_{j, 3}$, and $\tilde{E}_{j, 4}$ be sets in $\mathbb{R}^{2}$ determined as follows. For $j \leq\left\lfloor a_{t} / \delta_{t}\right\rfloor$,

$$
\begin{aligned}
\tilde{E}_{j, 1} & =\left\{\left((j-1) \delta_{t},(j-1) \delta_{t}+a_{t}\right) \times\left((j-1) \delta_{t}+a_{t}, \infty\right)\right\}, \\
\tilde{E}_{j, 2} & =\left\{\left((j-1) \delta_{t}+a_{t}, \infty\right) \times\left((j-1) \delta_{t},(j-1) \delta_{t}+a_{t}\right)\right\} .
\end{aligned}
$$

For $j \geq\left\lfloor a_{t} / \delta_{t}\right\rfloor+1$, let $\tilde{E}_{j, 1}$ and $\tilde{E}_{j, 2}$ be as above, and let

$$
\begin{aligned}
\tilde{E}_{j, 3} & =\left\{\left((j-1) \delta_{t},(j-1) \delta_{t}+a_{t}\right) \times\left(0,(j-1) \delta_{t}-a_{t}\right)\right\}, \\
\tilde{E}_{j, 4} & =\left\{\left(0,(j-1) \delta_{t}-a_{t}\right) \times\left((j-1) \delta_{t},(j-1) \delta_{t}+a_{t}\right)\right\} .
\end{aligned}
$$

Direct calculation shows that

$$
\begin{aligned}
V\left(\cup_{i=1}^{4} \tilde{E}_{j, i}\right)= & \iint_{\cup_{i=1}^{4} \tilde{E}_{i}} f_{2}(x, y) \mathrm{d} x \mathrm{~d} y \\
< & \int_{-\infty}^{\infty} \int_{(j-1) \delta_{t}}^{(j-1) \delta_{t}+a_{t}} f_{2}(x, y) \mathrm{d} x \mathrm{~d} y+\int_{(j-1) \delta_{t}}^{(j-1) \delta_{t}+a_{t}} \int_{-\infty}^{\infty} f_{2}(x, y) \mathrm{d} x \mathrm{~d} y \\
& +\int_{-\infty}^{\infty} \int_{(j-1) \delta_{t}}^{(j-1) \delta_{t}+a_{t}} f_{2}(x, y) \mathrm{d} x \mathrm{~d} y+\int_{(j-1) \delta_{t}}^{(j-1) \delta_{t}+a_{t}} \int_{-\infty}^{\infty} f_{2}(x, y) \mathrm{d} x \mathrm{~d} y \\
= & 4 a_{t} \quad \text { (see the detailed proof of (22) in Section 5). }
\end{aligned}
$$

For each $j, \cup_{i=1}^{4} \tilde{E}_{j, i}$ is the region of $A\left(Z_{j}^{-}\left(a_{t}\right)\right) \bigcap \cup_{i \notin D_{j}} A\left(Z_{i}^{-}\left(a_{t}\right)\right)$. Therefore, under the occurrence of the event of $\tilde{\mathcal{E}}: \Pi_{2}^{*} \bigcap\left\{\tilde{E}_{j, 1} \cup \tilde{E}_{j, 2} \cup \tilde{E}_{j, 3} \cup \tilde{E}_{j, 4}\right\}=\varnothing, Z_{j}^{-}\left(a_{t}\right)$ and $\sum_{i \notin \mathcal{D}_{j}} Z_{i}^{-}\left(a_{t}\right)$ are determined by the realizations of disjoint regions in the two-dimentional Poisson process, 
and are therefore independent. That is, $\left(\sum_{i \notin \mathcal{D}_{j}} Z_{i}^{-}\left(a_{t}\right) \mid \tilde{\mathcal{E}}\right)$ and $\left(Z_{j}^{-}\left(a_{t}\right) \mid \tilde{\mathcal{E}}\right)$ are independent. Therefore, the variation distance

$$
\begin{aligned}
& d\left(\left(\sum_{i \notin \mathcal{D}_{j}} Z_{i}^{-}\left(a_{t}\right)\left|Z_{j}^{-}\left(a_{t}\right)=1\right| \tilde{\mathcal{E}}\right),\left(\sum_{i \notin \mathcal{D}_{j}} Z_{i}^{-}\left(a_{t}\right) \mid \tilde{\mathcal{E}}\right)\right) \\
& \quad \equiv d\left(\left(\left(\sum_{i \notin \mathcal{D}_{j}} Z_{i}^{-}\left(a_{t}\right) \mid \tilde{\mathcal{E}}\right) \mid\left(Z_{j}^{-}\left(a_{t}\right)=1 \mid \tilde{\mathcal{E}}\right)\right),\left(\sum_{i \notin D_{j}} Z_{i}^{-}\left(a_{t}\right) \mid \tilde{\mathcal{E}}\right)\right) \\
& \quad=0 .
\end{aligned}
$$

Let $\tilde{\mathcal{E}}^{\mathrm{c}}$ denote the complement of $\tilde{\mathcal{E}}$. We have

$$
\begin{aligned}
& d\left(\left(\sum_{i \notin \mathcal{D}_{j}} Z_{i}^{-}\left(a_{t}\right) \mid Z_{j}^{-}\left(a_{t}\right)=1\right), \sum_{i \notin \mathcal{D}_{j}} Z_{i}^{-}\left(a_{t}\right)\right) \\
& \leq d\left(\left(\sum_{i \notin \mathcal{D}_{j}} Z_{i}^{-}\left(a_{t}\right)\left|Z_{j}^{-}\left(a_{t}\right)=1\right| \tilde{\mathcal{E}}\right),\left(\sum_{i \notin D_{j}} Z_{i}^{-}\left(a_{t}\right) \mid \tilde{\mathcal{E}}\right)\right) \operatorname{Pr}\{\tilde{\mathcal{E}}\} \\
& \quad+d\left(\left(\sum_{i \notin D_{j}} Z_{i}^{-}\left(a_{t}\right)\left|Z_{j}^{-}\left(a_{t}\right)=1\right| \tilde{\mathcal{E}}^{\mathrm{c}}\right),\left(\sum_{i \notin D_{j}} Z_{i}^{-}\left(a_{t}\right) \mid \tilde{\mathcal{E}}^{\mathrm{c}}\right)\right) \operatorname{Pr}\left\{\tilde{\mathcal{E}}^{\mathrm{c}}\right\} \\
& \quad \leq \operatorname{Pr}\left\{\tilde{\mathcal{E}}^{\mathrm{c}}\right\} \\
& \leq \operatorname{Pr}\left\{\Pi_{2}^{*} \cap\left\{\tilde{E}_{1} \cup \tilde{E}_{2} \cup \tilde{E}_{3} \cup \tilde{E}_{4}\right\} \neq \varnothing\right\} \\
& =1-\exp \left(-V\left(\cup_{i=1}^{4} \tilde{E}_{j, i}\right)\right) \\
& =4 a_{t}(1+o(1)) ;
\end{aligned}
$$

and it follows that

$$
\begin{aligned}
v_{3} & =2 \sum_{j=1}^{\left\lfloor(t-\sqrt[r]{x / t}) / \delta_{t}\right\rfloor} \mathrm{E}\left[Z_{j}^{-}\left(a_{t}\right)\right] d\left(\left(\sum_{i \notin D_{j}} Z_{i}^{-}\left(a_{t}\right) \mid Z_{j}^{-}\left(a_{t}\right)=1\right), \sum_{i \notin D_{j}} Z_{i}^{-}\left(a_{t}\right)\right) \\
& \leq 2\left(\sum_{j=1}^{\left\lfloor(t-\sqrt[r]{x / t}) / \delta_{t}\right\rfloor} \mathrm{E}\left[Z_{j}^{-}\left(a_{t}\right)\right]\right) 4 a_{t}(1+o(1)) \\
& =8 \alpha_{t} a_{t}(1+o(1)) \\
& \rightarrow 0
\end{aligned}
$$

as $\lim _{t \rightarrow \infty} \alpha_{t}=\alpha$ and $\lim _{t \rightarrow \infty} a_{t}=0$. Therefore, the Poisson law with parameter $\alpha$ for $n_{t}^{-}\left(a_{t}\right)$ is established.

\section{Details of estimates}

In this section the proof of the estimates of Section 3 will be elaborated.

Proof of Lemma 1. Some simple maniputations give

$$
\int_{0}^{z} \int_{-\infty}^{\infty} f_{2}(x, y) \mathrm{d} x \mathrm{~d} y=\int_{0}^{z} \int_{-\infty}^{\infty}\left(\int_{-\infty}^{\infty} g(x-s) g(y-s) \mathrm{d} x\right) \mathrm{d} s \mathrm{~d} y=z,
$$


and similarly for $\int_{-\infty}^{\infty} \int_{0}^{z} f_{2}(x, y) \mathrm{d} x \mathrm{~d} y=z$. Since

$$
\begin{aligned}
V\left(A_{1}(0, z)\right) & =\int_{0}^{z} \int_{0}^{z} f_{2}(x, y) \mathrm{d} x \mathrm{~d} y \\
& =2 \int_{0}^{z} \int_{0}^{x} f_{2}(x-y, 0) \mathrm{d} y \mathrm{~d} x \quad \text { (since } f_{2}(x, y)=f_{2}(y, x) \text { and } \\
& =2 \int_{0}^{z} \int_{0}^{x} f_{2}(s, 0) \mathrm{d} s \mathrm{~d} x \\
& =2 \int_{0}^{z} \int_{s}^{z} f_{2}(s, 0) \mathrm{d} x \mathrm{~d} x \\
& =2 \int_{0}^{z}(z-s) f_{2}(s, 0) \mathrm{d} s \\
& =z-2 \int_{0}^{\infty} s f_{2}(s, 0) \mathrm{d} s+2 \int_{z}^{\infty}(s-z) f_{2}(s, 0) \mathrm{d} s \\
& =z-\int_{-\infty}^{\infty}|s| f_{2}(s, 0) \mathrm{d} s+2 \int_{z}^{\infty}(s-z) f_{2}(s, 0) \mathrm{d} s \\
& =z-\int_{-\infty}^{\infty} \int_{-\infty}^{\infty}|u-v| g(u) g(v) \mathrm{d} u \mathrm{~d} v+2 \int_{z}^{\infty}(s-z) f_{2}(s, 0) \mathrm{d} s
\end{aligned}
$$

we have

$$
\begin{aligned}
2 z & =\int_{-\infty}^{\infty} \int_{0}^{z} f_{2}(x, y) \mathrm{d} x \mathrm{~d} y+\int_{0}^{z} \int_{-\infty}^{\infty} f_{2}(x, y) \mathrm{d} x \mathrm{~d} y \quad \text { (from (22)) } \\
& =V\left(A_{2}(0, z)\right)+2 V\left(A_{1}(0, z)\right) \quad\left(\text { from the defintions of } A_{1} \text { and } A_{2} \text { in Figure } 1\right) .
\end{aligned}
$$

Therefore, where $\int|s| g(s) \mathrm{d} s<\infty$, we have

$$
\begin{gathered}
\lim _{z \rightarrow \infty}\left(z-V\left(A_{1}(0, z)\right)\right)=\int_{-\infty}^{\infty} \int_{-\infty}^{\infty}|u-v| g(u) g(v) \mathrm{d} u \mathrm{~d} v \\
\lim _{z \rightarrow \infty} V\left(A_{2}(0, z)\right)=2 \int_{-\infty}^{\infty} \int_{-\infty}^{\infty}|u-v| g(u) g(v) \mathrm{d} u \mathrm{~d} v .
\end{gathered}
$$

Now, we have

$\operatorname{Pr}\left\{\right.$ there are at most $k$ points of $\Pi^{(2)}$ in $\left.(0, z)\right\}$

$$
=\sum_{j=0}^{\lfloor k / 2\rfloor} \operatorname{Pr}\left\{\left\|A_{1}(0, z)\right\|=j ;\left\|A_{2}(0, z)\right\| \leq k-2 j\right\}
$$

and since $A_{1}(0, z)$ and $A_{2}(0, z)$ are disjoint regions, and $\Pi_{2}^{\star}$ is a two-dimensional Poisson process, we have

$$
=\sum_{j=0}^{\lfloor k / 2\rfloor} \mathrm{e}^{-V\left(A_{1}(0, z)\right)} \frac{\left[V\left(A_{1}(0, z)\right)\right]^{j}}{j !} \mathrm{e}^{-V\left(A_{2}(0, z)\right)}\left(\sum_{i=0}^{k-2 j} \frac{\left[V\left(A_{2}(0, z)\right)\right]^{i}}{i !}\right)
$$




$$
\begin{aligned}
= & \mathrm{e}^{-V\left(A_{1}(0, z)\right)} \frac{\left[V\left(A_{1}(0, z)\right)\right]^{\lfloor k / 2\rfloor}}{\lfloor k / 2\rfloor !} \mathrm{e}^{-V\left(A_{2}(0, z)\right)} I_{2}(k) \quad\left(\text { for } I_{2}(k)\right. \text { as defined in (8)) } \\
& +\sum_{j=0}^{\lfloor k / 2\rfloor-1} \mathrm{e}^{-V\left(A_{1}(0, z)\right)} \frac{\left[V\left(A_{1}(0, z)\right)\right]^{j}}{j !} \mathrm{e}^{-V\left(A_{2}(0, z)\right)}\left(\sum_{i=0}^{k-2 j} \frac{\left[V\left(A_{2}(0, z)\right)\right]^{i}}{i !}\right) \\
= & \exp \left\{-\left(V\left(A_{1}(0, z)\right)+V\left(A_{2}(0, z)\right)\right)\right\} \frac{\left[V\left(A_{1}(0, z)\right)\right]^{\lfloor k / 2\rfloor}}{\lfloor k / 2\rfloor !} I_{2}(k) \\
& +\operatorname{smaller-order~terms~of~} z, \text { and using }(23) \text { and }(24) \\
= & \exp \left\{-\left(z+\int_{-\infty}^{\infty} \int_{-\infty}^{\infty}|u-v| g(u) g(v) \mathrm{d} u \mathrm{~d} v\right)\right\} \\
& \times \frac{\left[z-\int_{-\infty}^{\infty} \int_{-\infty}^{\infty}|u-v| g(u) g(v) \mathrm{d} u \mathrm{~d} v\right]^{\lfloor k / 2\rfloor}}{\lfloor k / 2\rfloor !} \\
& \times I_{2}(k)(1+o(1)) .
\end{aligned}
$$

Proof of Lemma 2. Lemma 2 is proved by evaluating the requisite events of the two-dimensional Poisson process $\Pi_{2}^{*}$. Observe that

$$
\begin{aligned}
\operatorname{Pr}\left\{\left\|\left(\triangle_{t}, \triangle_{t}+b_{t}\right)\right\| \leq r\right\}-\operatorname{Pr}\left\{\left\|\left(0, \triangle_{t}+b_{t}\right)\right\| \leq r\right\} \\
=\operatorname{Pr}\left\{\left\|\left(\triangle_{t}, \triangle_{t}+b_{t}\right)\right\| \leq r,\left\|\left(0, \triangle_{t}+b_{t}\right)\right\|>r\right\} \\
=\sum_{k=1}^{r} \operatorname{Pr}\left\{\left\|\left(0, \triangle_{t}\right)\right\|=k ;\left\|\left(\triangle_{t}, \triangle_{t}+b_{t}\right)\right\| \leq r-k\right\} \\
=\operatorname{Pr}\left\{\left\|\left(0, \triangle_{t}\right)\right\|=1 ;\left\|\left(\triangle_{t}, \triangle_{t}+b_{t}\right)\right\| \leq r-1\right\} \\
\quad+\sum_{k=2}^{r} \operatorname{Pr}\left\{\left\|\left(0, \triangle_{t}\right)\right\|=k ;\left\|\left(\triangle_{t}, \triangle_{t}+b_{t}\right)\right\| \leq r-k\right\} .
\end{aligned}
$$

Therefore,

$$
\begin{array}{r}
\operatorname{Pr}\left\{\left\|\left(0, \triangle_{t}\right)\right\|=1 ;\left\|\left(\triangle_{t}, \triangle_{t}+b_{t}\right)\right\| \leq r-1\right\} \\
=\operatorname{Pr}\left\{\left\|\left(\triangle_{t}, \triangle_{t}+b_{t}\right)\right\| \leq r\right\}-\operatorname{Pr}\left\{\left\|\left(0, \triangle_{t}+b_{t}\right)\right\| \leq r\right\} \\
\quad-\sum_{k=2}^{r} \operatorname{Pr}\left\{\left\|\left(0, \triangle_{t}\right)\right\|=k ;\left\|\left(\triangle_{t}, \triangle_{t}+b_{t}\right)\right\| \leq r-k\right\}
\end{array}
$$

applying (25) for $z=b_{t}=\ln t+\lfloor(r-1) / 2\rfloor \ln \ln t+x$ and $z=\triangle_{t}+b_{t}$, and also knowing that $\operatorname{Pr}\left\{\left\|\left(0, \triangle_{t}\right)\right\|=k ;\left\|\left(\triangle_{t}, \triangle_{t}+b_{t}\right)\right\| \leq r-k\right\} \leq O\left(\triangle_{t}^{k}\right) O\left(\exp \left(-b_{t}\right) b_{t}^{\lfloor(r-k) / 2\rfloor}\right)$, we obtain

$$
=\frac{\triangle_{t} \exp \left\{-\left(x+\int_{-\infty}^{\infty} \int_{-\infty}^{\infty}|u-v| g(u) g(v) \mathrm{d} u \mathrm{~d} v\right)\right\}}{\lfloor(r-1) / 2\rfloor ! t}(1+o(1))
$$

$o(1) \rightarrow 0$ as $t \rightarrow \infty$ 
Proof of Lemma 3. First we consider the following two-dimensional disjoint sets:

$$
\begin{aligned}
& C_{1}=\left\{(-\infty, 0) \times\left(0, \triangle_{t}\right)\right\} \cup\left\{\left(0, \triangle_{t}\right) \times(-\infty, 0)\right\}, \\
& C_{2}=\left\{\left(\triangle_{t}+b_{t}, \infty\right) \times\left(0, \triangle_{t}\right)\right\} \cup\left\{\left(0, \triangle_{t}\right) \times\left(\triangle_{t}+b_{t}, \infty\right)\right\}, \\
& C_{3}=\left\{\left(\triangle_{t}, \triangle_{t}+b_{t}\right) \times\left(0, \triangle_{t}\right)\right\} \cup\left\{\left(0, \triangle_{t}\right) \times\left(\triangle_{t}, \triangle_{t}+b_{t}\right)\right\}, \\
& C_{4}=\left\{\left(0, \triangle_{t}\right) \times\left(0, \triangle_{t}\right)\right\} .
\end{aligned}
$$

Consider the sets $A\left(\triangle_{t}, b_{t}+\triangle_{t}\right), A_{1}\left(\triangle_{t}, b_{t}+\triangle_{t}\right)$, and $A_{2}\left(\triangle_{t}, b_{t}+\triangle_{t}\right)$ as defined in Figure 1 by setting $a=\triangle_{t}$ and $b=b_{t}+\triangle_{t}$. It should be noted that $C_{3}$ is a subset of $A_{2}\left(\triangle_{t}, b_{t}+\triangle_{t}\right)$. Based on (22), we have

$$
\begin{aligned}
V\left(C_{1}\right)+V\left(C_{2}\right)+V\left(C_{3}\right)+2 V\left(C_{4}\right) & =\int_{0}^{\triangle_{t}} \int_{-\infty}^{\infty} f_{2}(x, y) \mathrm{d} x \mathrm{~d} y+\int_{-\infty}^{\infty} \int_{0}^{\triangle_{t}} f_{2}(x, y) \mathrm{d} x \mathrm{~d} y \\
& =2 \triangle_{t} .
\end{aligned}
$$

Also,

$$
\begin{aligned}
V\left(C_{4}\right) & =\int_{0}^{\triangle_{t}} \int_{0}^{\triangle_{t}} f_{2}(x, y) \mathrm{d} x \mathrm{~d} y \\
& =f_{2}(0,0) \triangle_{t}^{2}(1+o(1)) \quad \text { with } f_{2}(0,0)=\int_{-\infty}^{\infty} g^{2}(\eta) \mathrm{d} \eta
\end{aligned}
$$

and

$$
V\left(C_{3}\right)=2 \int_{0}^{\triangle_{t}} \int_{\triangle_{t}}^{\triangle_{t}+b_{t}} f_{2}(x, y) \mathrm{d} x \mathrm{~d} y=2 \int_{0}^{\triangle_{t}} \int_{\triangle_{t}}^{\triangle_{t}+b_{t}} f_{2}(x-y, 0) \mathrm{d} x \mathrm{~d} y .
$$

Since, for each fixed $y, 0<y<\triangle_{t}$,

$$
\int_{\triangle_{t}}^{b_{t}} f_{2}(x, 0) \mathrm{d} x<\int_{\Delta_{t}}^{\Delta_{t}+b_{t}} f_{2}(x-y, 0) \mathrm{d} x<\int_{0}^{\triangle_{t}+b_{t}} f_{2}(x, 0) \mathrm{d} x,
$$

we conclude, after integrating over $y$ from 0 to $\triangle_{t}$, that

$$
2 \triangle_{t} \int_{\triangle_{t}}^{b_{t}} f_{2}(x, 0) \mathrm{d} x<V\left(C_{3}\right)<2 \triangle_{t} \int_{0}^{\triangle_{t}+b_{t}} f_{2}(x, 0) \mathrm{d} x
$$

which can be rewritten as

$$
\triangle_{t}\left(\int_{-b_{t}}^{b_{t}} f_{2}(x, 0) \mathrm{d} x-\int_{-\triangle_{t}}^{\triangle_{t}} f_{2}(x, 0) \mathrm{d} x\right)<V\left(C_{3}\right)<\triangle_{t} \int_{-\left(\Delta_{t}+b_{t}\right)}^{\triangle_{t}+b_{t}} f_{2}(x, 0) \mathrm{d} x,
$$

and therefore,

$$
V\left(C_{3}\right)=\triangle_{t}(1+o(1))
$$

Owing to (27)-(29), we have

$$
V\left(C_{1} \cup C_{2}\right)=2 \triangle_{t}-V\left(C_{3}\right)-2 V\left(C_{4}\right)=\triangle_{t}(1+o(1)) .
$$

According to (23) and (24), in the proof of Lemma 1, with $z=b_{t}$, as $t \rightarrow \infty$,

$$
V\left(A_{1}\left(\triangle_{t}, b_{t}+\triangle_{t}\right)\right)=b_{t}-\int_{-\infty}^{\infty} \int_{-\infty}^{\infty}|u-v| g(u) g(v) \mathrm{d} u \mathrm{~d} v+o(1) .
$$


Consider $\left\{C_{i}\right\}_{i=1}^{4}$ as defined in (26) and $A_{1}\left(\triangle_{t}, \triangle_{t}+b_{t}\right)$ and $A_{2}\left(\triangle_{t}, \triangle_{t}+b_{t}\right)$ as defined in Figure 1. The probability of the event $\left\{\left\|\left(0, \triangle_{t}\right)\right\| \geq 2,\left\|\left(\triangle_{t}, \triangle_{t}+b_{t}\right)\right\| \leq r-1\right\}$ can be bounded as follows.

$$
\begin{aligned}
& \operatorname{Pr}\left\{\left\|\left(0, \triangle_{t}\right)\right\| \geq 2,\left\|\left(\triangle_{t}, \triangle_{t}+b_{t}\right)\right\| \leq r-1\right\} \\
&= \operatorname{Pr}\left\{\left\|C_{1} \cup C_{2} \cup C_{3}\right\|+2\left\|C_{4}\right\| \geq 2 ; 2\left\|A_{1}\left(\triangle_{t}, \triangle_{t}+b_{t}\right)\right\|\right. \\
&\left.+\left\|A_{2}\left(\triangle_{t}, \triangle_{t}+b_{t}\right)\right\| \leq r-1\right\} \\
&= \sum_{j=0}^{\lfloor(r-1) / 2\rfloor} \operatorname{Pr}\left\{\left\|C_{1} \cup C_{2} \cup C_{3}\right\|+2\left\|C_{4}\right\| \geq 2 ;\left\|A_{1}\left(\triangle_{t}, \triangle_{t}+b_{t}\right)\right\|=j ;\right. \\
&\left.\left\|A_{2}\left(\triangle_{t}, \triangle_{t}+b_{t}\right)\right\| \leq r-1-2 j\right\} \\
& \leq \sum_{j=0}^{\lfloor(r-1) / 2\rfloor} \operatorname{Pr}\left\{\left\|C_{1} \cup C_{2} \cup C_{3}\right\|+2\left\|C_{4}\right\| \geq 2 ;\left\|A_{1}\left(\triangle_{t}, \triangle_{t}+b_{t}\right)\right\|=j\right\},
\end{aligned}
$$

since $C_{1}, C_{2}, C_{3}, C_{4}$, and $A_{1}\left(\triangle_{t}, \triangle_{t}+b_{t}\right)$ are all disjoint regions, we have the bound

$$
\begin{aligned}
< & \left(\operatorname{Pr}\left\{\left\|C_{1} \cup C_{2} \cup C_{3}\right\| \geq 2\right\}+\operatorname{Pr}\left\{\left\|C_{4}\right\| \geq 1\right\}\right) \\
& \times\left(\sum_{j=0}^{\lfloor(r-1) / 2\rfloor} \operatorname{Pr}\left\{\left\|A_{1}\left(\triangle_{t}, \triangle_{t}+b_{t}\right)\right\|=j\right\}\right),
\end{aligned}
$$

and, according to the Poisson law and evaluations of $V\left(C_{1} \cup C_{2} \cup C_{3}\right), V\left(C_{4}\right)$, and $V\left(A_{1}\left(\triangle_{t}\right.\right.$, $\left.\left.\triangle_{t}+b_{t}\right)\right)$ in (28)-(31), we obtain

$$
\begin{aligned}
& =O\left(\triangle_{t}^{2}\right) O\left(\mathrm{e}^{-b_{t}} \frac{b_{t}^{\lfloor(r-1) / 2\rfloor}}{\lfloor(r-1) / 2\rfloor}\right) \\
& =O\left(\frac{\triangle_{t}^{2}}{t}\right) .
\end{aligned}
$$

The probability of the event $\left\{\left\|\left(0, \triangle_{t}\right)\right\|=1,\left\|\left(0, b_{t}\right)\right\| \leq r,\left\|\left(0, \triangle_{t}+b_{t}\right)\right\| \geq r+1\right\}$ can be bounded as follows.

$$
\begin{aligned}
& \operatorname{Pr}\left\{\left\|\left(0, \triangle_{t}\right)\right\|=1,\left\|\left(0, b_{t}\right)\right\| \leq r,\left\|\left(0, \triangle_{t}+b_{t}\right)\right\| \geq r+1\right\} \\
& \quad<\operatorname{Pr}\left\{\left\|\left(0, \triangle_{t}\right)\right\|=1,\left\|\left(\triangle_{t}, b_{t}\right)\right\| \leq r-1,\left\|\left(b_{t}, \triangle_{t}+b_{t}\right)\right\| \geq 1\right\},
\end{aligned}
$$

since $A_{1}\left(\triangle_{t}, b_{t}\right)$ is disjoint from both $A\left(0, \triangle_{t}\right)$ and $A\left(b_{t}, b_{t}+\triangle_{t}\right)$, we have the bound

$$
\begin{aligned}
\leq & \operatorname{Pr}\left\{\left\|\left(0, \triangle_{t}\right)\right\|=1,\left\|\left(b_{t}, \triangle_{t}+b_{t}\right)\right\| \geq 1\right\} \operatorname{Pr}\left\{\left\|A_{1}\left(\triangle_{t}, b_{t}\right)\right\| \leq\left\lfloor\frac{r-1}{2}\right\rfloor\right\} \\
= & \left(\operatorname{Pr}\left\{\left\|\left(0, \triangle_{t}\right)\right\|=1\right\}-\operatorname{Pr}\left\{\left\|\left(0, \triangle_{t}\right)\right\|=1,\left\|\left(b_{t}, \triangle_{t}+b_{t}\right)\right\|=0\right\}\right) \\
& \times \operatorname{Pr}\left\{\left\|A_{1}\left(\triangle_{t}, b_{t}\right)\right\| \leq\left\lfloor\frac{r-1}{2}\right\rfloor\right\},
\end{aligned}
$$

then, according to the Poisson law, we obtain

$$
=O\left(\mathrm{e}^{-2 \triangle_{t}}\left(2 \triangle_{t}\right)-\mathrm{e}^{-2 \triangle_{t}}\left(2 \triangle_{t}\right) \mathrm{e}^{-2 \triangle_{t}}\right) O\left(\mathrm{e}^{-b_{t}} \frac{b_{t}^{\lfloor(r-1) / 2\rfloor}}{\lfloor(r-1) / 2\rfloor !}\right)
$$




$$
\begin{aligned}
& =O\left(\triangle_{t}^{2}\right) O\left(\mathrm{e}^{-\ln t}\right) \\
& =O\left(\frac{\triangle_{t}^{2}}{t}\right) .
\end{aligned}
$$

This completes the proof.

Proof of Lemma 4. If $r=2 p+1$ and $p$ is a nonnegative integer, we will verify the estimates, (35), (36), and (37), below, of $\mathrm{E}\left[Z_{j}^{+}\left(b_{t}\right) Z_{i}^{+}\left(b_{t}\right)\right], j \neq i$, which is involved in the calculation of the error parameter $u_{2}$ of the Chen-Stein method in (1), where the neighborhood subset $B_{i}$ is specified as

$$
\begin{aligned}
B_{i}= & \left\{j:|j-i|<\left\lfloor\frac{2 b_{t}}{\triangle_{t}}\right\rfloor\right\} \\
= & \left\{j:|j-i| \leq\left\lfloor\frac{\ln \ln t}{\triangle_{t}}\right\rfloor\right\} \cup\left\{j:\left\lfloor\frac{\ln \ln t}{\triangle_{t}}\right\rfloor<|j-i| \leq\left\lfloor\frac{b_{t}}{\triangle_{t}}\right\rfloor\right\} \\
& \cup\left\{j:\left\lfloor\frac{b_{t}}{\triangle_{t}}\right\rfloor<|j-i| \leq\left\lfloor\frac{2 b_{t}}{\triangle_{t}}\right\rfloor\right\} \\
= & \text { (I) }+ \text { (II) }+ \text { (III). }
\end{aligned}
$$

For the case in which $p=0$ (1-scan case), we have, for $1 \leq|i-j| \leq\left\lfloor b_{t} / \triangle_{t}\right\rfloor$,

$$
\begin{aligned}
& \mathrm{E}\left[Z_{j}^{+}\left(b_{t}\right) Z_{i}^{+}\left(b_{t}\right)\right]=\mathrm{E}\left[Z_{1}^{+}\left(b_{t}\right) Z_{i-j+1}^{+}\left(b_{t}\right)\right] \\
& =\operatorname{Pr}\left\{\left\|\left(0, \triangle_{t}\right)\right\|=1,\left\|\left(\triangle_{t}, \triangle_{t}+b_{t}\right)\right\|=0,\right. \\
& \left\|\left((i-j) \triangle_{t},(i-j+1) \triangle_{t}\right)\right\|=1 \text {, } \\
& \left.\left\|\left((i-j+1) \triangle_{t},(i-j+1) \triangle_{t}+b_{t}\right)\right\|=0\right\} \quad \text { (directly from the defini- } \\
& \text { tions of } Z_{j}^{+} \text {and } Z_{i}^{+} \text {), }
\end{aligned}
$$

since $\left\|\left((i-j) \triangle_{t},(i-j+1) \triangle_{t}\right)\right\|=1$ and $\left\|\left(\triangle_{t}, \triangle_{t}+b_{t}\right)\right\|=0$ are disjoint events for $1 \leq|i-j| \leq\left\lfloor b_{t} / \triangle_{t}\right\rfloor$ the last equation equals 0 .

For $\left\lfloor b_{t} / \Delta_{t}\right\rfloor+1 \leq|j-i| \leq\left\lfloor 2 b_{t} / \Delta_{t}\right\rfloor$, we have

$$
\begin{aligned}
\mathrm{E}\left[Z_{j}^{+}\left(b_{t}\right) Z_{i}^{+}\left(b_{t}\right)\right]= & \mathrm{E}\left[Z_{1}^{+}\left(b_{t}\right) Z_{i-j+1}^{+}\left(b_{t}\right)\right] \\
= & \operatorname{Pr}\left\{\left\|\left(0, \triangle_{t}\right)\right\|=1,\left\|\left(\triangle_{t}, \triangle_{t}+b_{t}\right)\right\|=0,\right. \\
& \left\|\left((i-j) \triangle_{t},(i-j+1) \triangle_{t}\right)\right\|=1, \\
& \left.\left\|\left((i-j+1) \triangle_{t},(i-j+1) \triangle_{t}+b_{t}\right)\right\|=0\right\} .
\end{aligned}
$$

Because of the disjoint nature of $A_{1}(a, b)$ to other regions of $A(c, d)$ in the two-dimensional space when $(a, b)$ and $(c, d)$ are nonoverlapping intervals of the line, we have

$$
\begin{aligned}
\leq & \operatorname{Pr}\left\{\left\|\left(0, \triangle_{t}\right)\right\|=1,\left\|\left((i-j) \triangle_{t},(i-j+1) \triangle_{t}\right)\right\|=1,\right\} \\
& \times \operatorname{Pr}\left\{\left\|A_{1}\left(\triangle_{t}, \triangle_{t}+b_{t}\right)\right\|=0\right\} \\
& \times \operatorname{Pr}\left\{\left\|A_{1}\left((i-j+1) \triangle_{t},(i-j+1) \triangle_{t}+b_{t}\right)\right\|=0,\right\} \\
= & O\left(\triangle_{t}^{2}\right) O\left(\mathrm{e}^{-b_{t}}\right) O\left(\mathrm{e}^{-b_{t}}\right) \\
\leq & O\left(\frac{\triangle^{2}}{t^{2}}\right) .
\end{aligned}
$$


To sum up, from (33) and (34) we obtain, when $p=0(r=1)$,

$$
\sum_{j \in B_{i}, j \neq i} \mathrm{E}\left[Z_{i}^{+}\left(b_{t}\right) Z_{j}^{+}\left(b_{t}\right)\right] \leq 2 \frac{b_{t}}{\triangle_{t}} O\left(\frac{\triangle_{t}^{2}}{t^{2}}\right) \leq O\left(\frac{\ln t}{t^{3}}\right)
$$

For the case in which $p \geq 1$ ( $r \geq 3$ ), the bounds of (I), (II), and (III) in (32) are evaluated next. For (I) in (32) and $1 \leq|i-j| \leq\left\lfloor\ln \ln t / \triangle_{t}\right\rfloor$,

$$
\begin{aligned}
\mathrm{E}\left[Z_{j}^{+}\left(b_{t}\right) Z_{i}^{+}\left(b_{t}\right)\right]= & \left.\mathrm{E}\left[Z_{1}^{+}\left(b_{t}\right) Z_{i-j+1}^{+}\left(b_{t}\right)\right] \quad \text { (by the stationary property of } \Pi_{1}^{(2)}\right) \\
< & \operatorname{Pr}\left\{\left\|\left(0, \triangle_{t}\right)\right\|=1,\left\|\left((i-j) \triangle_{t},(i-j+1) \triangle_{t}\right)\right\|=1,\right. \\
& \left.\quad\left\|\left(\triangle_{t}, \triangle_{t}+b_{t}\right) \backslash\left((i-j) \triangle_{t},(i-j+1) \triangle_{t}\right)\right\| \leq r-2\right\} \\
\leq & \operatorname{Pr}\left\{\left\|\left(0, \triangle_{t}\right)\right\|=1,\left\|\left((i-j) \triangle_{t},(i-j+1) \triangle_{t}\right)\right\|=1\right\} \\
& \times \operatorname{Pr}\left\{\left\|A_{1}\left(\triangle_{t}, \triangle_{t}+b_{t}\right) \backslash A\left((i-j) \triangle_{t},(i-j+1) \triangle_{t}\right)\right\| \leq\left\lfloor\frac{r-2}{2}\right\rfloor\right\} \\
= & O\left(\triangle_{t}^{2}\right) O\left(\mathrm{e}^{-b_{t}} \frac{b_{t}^{\lfloor(r-2) / 2\rfloor !}}{\lfloor(r-2) / 2\rfloor !}\right) \\
= & O\left(\frac{\triangle_{t}^{2}}{t \ln t}\right) .
\end{aligned}
$$

For (II) in (32) and $\left\lfloor\ln \ln t / \triangle_{t}\right\rfloor+1 \leq|i-j| \leq\left\lfloor b_{t} / \triangle_{t}\right\rfloor$,

$$
\begin{aligned}
\mathrm{E}\left[Z_{j}^{+}\left(b_{t}\right) Z_{i}^{+}\left(b_{t}\right)\right]= & \mathrm{E}\left[Z_{1}^{+}\left(b_{t}\right) Z_{i-j+1}^{+}\left(b_{t}\right)\right] \\
\leq & \operatorname{Pr}\left\{\left\|\left(0, \triangle_{t}\right)\right\|=1,\left\|\left((i-j) \triangle_{t},(i-j+1) \triangle_{t}\right)\right\|=1,\right. \\
& \left\|\left(\triangle_{t}, \triangle_{t}+b_{t}\right) \backslash\left((i-j) \triangle_{t},(i-j+1) \triangle_{t}\right)\right\| \leq r-2, \\
& \left.\left\|\left(\triangle_{t}+b_{t}, \triangle_{t}+b_{t}+\ln \ln t\right)\right\| \leq r-1\right\} \\
\leq & \operatorname{Pr}\left\{\left\|\left(0, \triangle_{t}\right)\right\|=1,\left\|\left((i-j) \triangle_{t},(i-j+1) \triangle_{t}\right)\right\|=1\right\} \\
& \times \operatorname{Pr}\left\{\left\|A_{1}\left(\triangle_{t}, \triangle_{t}+b_{t}\right) \backslash A\left((i-j) \triangle_{t},(i-j+1) \triangle_{t}\right)\right\| \leq r-2\right\} \\
& \times \operatorname{Pr}\left\{\left\|A_{1}\left(\triangle_{t}+b_{t}, \triangle_{t}+b_{t}+\ln \ln t\right)\right\| \leq r-1\right\} \\
= & O\left(\triangle_{t}^{2}\right) O\left(\mathrm{e}^{-b_{t}} \frac{b_{t}^{\lfloor(r-2) / 2\rfloor}}{\lfloor(r-2) / 2\rfloor !}\right) O\left(\mathrm{e}^{-\ln \ln t} \frac{(\ln \ln t)^{\lfloor(r-1) / 2\rfloor}}{\lfloor(r-1) / 2\rfloor !}\right) \\
= & O\left(\frac{\triangle_{t}^{2}(\ln \ln t)^{\lfloor(r-1) / 2\rfloor}}{t(\ln t)^{2}}\right) .
\end{aligned}
$$

For (III) in (32) and $\left\lfloor b_{t} / \triangle_{t}\right\rfloor+1 \leq i-j \leq\left\lfloor 2 b_{t} / \triangle_{t}\right\rfloor$, using an argument similar to the one used above for the case in which $p=0$, we have

$$
\begin{aligned}
\mathrm{E}\left[Z_{j}^{+}\left(b_{t}\right) Z_{i}^{+}\left(b_{t}\right)\right]= & \mathrm{E}\left[Z_{1}^{+}\left(b_{t}\right) Z_{i-j+1}^{+}\left(b_{t}\right)\right] \\
=\operatorname{Pr}\{ & \left\|\left(0, \triangle_{t}\right)\right\|=1,\left\|\left(\triangle_{t}, \triangle_{t}+b_{t}\right)\right\| \leq r-1, \\
& \left\|\left((i-j) \triangle_{t},(i-j+1) \triangle_{t}\right)\right\|=1, \\
& \left.\left\|\left((i-j+1) \triangle_{t},(i-j+1) \triangle_{t}+b_{t}\right)\right\| \leq r-1\right\}
\end{aligned}
$$




$$
\begin{aligned}
\leq & \operatorname{Pr}\left\{\left\|\left(0, \triangle_{t}\right)\right\|=1,\left\|\left((i-j) \triangle_{t},(i-j+1) \triangle_{t}\right)\right\|=1,\right\} \\
& \times \operatorname{Pr}\left\{\left\|A_{1}\left(\triangle_{t}, \triangle_{t}+b_{t}\right)\right\| \leq r-1\right\} \\
& \times \operatorname{Pr}\left\{\left\|A_{1}\left((i-j+1) \triangle_{t},(i-j+1) \triangle_{t}+b_{t}\right)\right\| \leq r-1\right\} \\
= & O\left(\triangle_{t}^{2}\right) O\left(\mathrm{e}^{-b_{t}} \frac{b_{t}^{\lfloor(r-1) / 2\rfloor}}{\lfloor(r-1) / 2\rfloor !}\right) O\left(\mathrm{e}^{-b_{t}} \frac{b_{t}^{\lfloor(r-1) / 2\rfloor}}{\lfloor(r-1) / 2\rfloor !}\right) \\
= & O\left(\frac{\triangle_{t}^{2}}{t^{2}}\right) .
\end{aligned}
$$

Therefore, from (35), (36), and (37) we have, for $p \geq 1$,

$$
\begin{aligned}
\sum_{j \in B_{i}, j \neq i} \mathrm{E}\left[Z_{i}^{+}\left(b_{t}\right) Z_{j}^{+}\left(b_{t}\right)\right]= & \sum_{1 \leq|j-i| \leq\left\lfloor\ln \ln t / \triangle_{t}\right\rfloor} \mathrm{E}\left[Z_{i}^{+}\left(b_{t}\right) Z_{j}^{+}\left(b_{t}\right)\right] \\
& +\sum_{\left\lfloor\ln \ln t / \triangle_{t}\right\rfloor<|j-i| \leq\left\lfloor b_{t} / \triangle_{t}\right\rfloor} \mathrm{E}\left[Z_{i}^{+}\left(b_{t}\right) Z_{j}^{+}\left(b_{t}\right)\right] \\
& +\sum_{\left\lfloor b_{t} / \triangle_{t}\right\rfloor \leq|i-j| \leq\left\lfloor 2 b_{t} / \triangle_{t}\right\rfloor} \mathrm{E}\left[Z_{i}^{+}\left(b_{t}\right) Z_{j}^{+}\left(b_{t}\right)\right] \\
\leq & 2\left\lfloor\frac{\ln \ln t}{\triangle_{t}}\right\rfloor O\left(\frac{\triangle_{t}^{2}}{t \ln t}\right)+2\left\lfloor\frac{b_{t}}{\triangle_{t}}\right\rfloor O\left(\frac{\triangle_{t}^{2}(\ln \ln t)^{\lfloor(r-1) / 2\rfloor}}{t(\ln t)^{2}}\right) \\
& +2\left\lfloor\frac{2 b_{t}}{\triangle_{t}}\right\rfloor O\left(\frac{\triangle_{t}^{2}}{t^{2}}\right) .
\end{aligned}
$$

This completes the proof.

Proof of Lemma 5. For $y \rightarrow 0$,

$$
\begin{aligned}
& \operatorname{Pr}\{\|(0, y)\| \geq r+1\} \\
& =\sum_{j=0}^{\lfloor(r+1) / 2\rfloor} \operatorname{Pr}\left\{\left\|A_{1}(0, y)\right\|=j,\left\|A_{2}(0, y)\right\| \geq r+1-2 j\right\} \\
& \quad+\operatorname{Pr}\left\{\left\|A_{1}(0, y)\right\| \geq\left\lfloor\frac{r+1}{2}\right\rfloor+1\right\},
\end{aligned}
$$

then by substituting $V\left(A_{1}(0, y)\right)=f_{2}(0,0) y^{2}(1+o(1))(o(1) \rightarrow 0$ as $y \rightarrow 0)$, and $V\left(A_{2}(0, y)\right)=2 y-V\left(A_{1}(0, y)\right)$, we obtain

$$
\begin{aligned}
= & \sum_{j=0}^{\lfloor(r+1) / 2\rfloor}\left\{\exp \left(-\left\{f_{2}(0,0) y^{2}\right\}\right) \frac{\left(f_{2}(0,0) y^{2}\right)^{j}}{j !} \exp (-2 y) \frac{(2 y)^{r+1-2 j}}{(r+1-2 j) !}\right\} \\
& +\exp \left\{-f_{2}(0,0) y^{2}\right\} \frac{\left(f_{2}(0,0) y^{2}\right)^{\lfloor(r+1) / 2\rfloor+1}}{(\lfloor(r+1) / 2\rfloor+1) !}+\text { smaller-order terms of } y \\
= & \left\{\sum_{j=0}^{\lfloor(r+1) / 2\rfloor} \frac{f_{2}(0,0)^{j}}{j !} \frac{2^{r+1-2 j}}{(r+1-2 j) !}\right\} y^{r+1}+\text { smaller-order terms of } y .
\end{aligned}
$$


Also,

$$
\begin{aligned}
& \operatorname{Pr}\left\{\left\|\left(0, a_{t}\right)\right\| \geq r+1\right\}-\operatorname{Pr}\left\{\left\|\left(\delta_{t}, a_{t}\right)\right\| \geq r+1\right\} \\
& =\operatorname{Pr}\left\{\left\|\left(0, a_{t}\right)\right\| \geq r+1,\left\|\left(\delta_{t}, a_{t}\right)\right\| \leq r\right\} \\
& =\operatorname{Pr}\left\{\left\|\left(0, \delta_{t}\right)\right\| \geq 1,\left\|\left(\delta_{t}, a_{t}\right)\right\|=r\right\}+\sum_{j=0}^{r-1} \operatorname{Pr}\left\{\left\|\left(0, \delta_{t}\right)\right\| \geq r+1-j,\left\|\left(\delta_{t}, a_{t}\right)\right\|=j\right\} \\
& =\operatorname{Pr}\left\{\left\|\left(0, \delta_{t}\right)\right\|=1,\left\|\left(\delta_{t}, a_{t}\right)\right\|=r\right\}+\operatorname{Pr}\left\{\left\|\left(0, \delta_{t}\right)\right\| \geq 2,\left\|\left(\delta_{t}, a_{t}\right)\right\|=r\right\} \\
& \quad+\sum_{j=0}^{r-1} \operatorname{Pr}\left\{\left\|\left(0, \delta_{t}\right)\right\| \geq r+1-j,\left\|\left(\delta_{t}, a_{t}\right)\right\|=j\right\} .
\end{aligned}
$$

Therefore,

$$
\begin{aligned}
& \operatorname{Pr}\left\{\left\|\left(0, \delta_{t}\right)\right\|=1,\left\|\left(\delta_{t}, a_{t}\right)\right\|=r\right\} \\
& =\operatorname{Pr}\left\{\left\|\left(0, a_{t}\right)\right\| \geq r+1\right\}-\operatorname{Pr}\left\{\left\|\left(\delta_{t}, a_{t}\right)\right\| \geq r+1\right\} \\
& \quad-\operatorname{Pr}\left\{\left\|\left(0, \delta_{t}\right)\right\| \geq 2,\left\|\left(\delta_{t}, a_{t}\right)\right\|=r\right\}-\sum_{j=0}^{r-1} \operatorname{Pr}\left\{\left\|\left(0, \delta_{t}\right)\right\| \geq r+1-j,\left\|\left(\delta_{t}, a_{t}\right)\right\|=j\right\},
\end{aligned}
$$

then, using (38) for $y=a_{t}=\sqrt[r]{x / t}$ and $\delta_{t}=1 / t^{2}$, and knowing that $\operatorname{Pr}\left\{\left\|\left(0, \delta_{t}\right)\right\| \geq\right.$ 2, $\left.\left\|\left(\delta_{t}, a_{t}\right)\right\|=r\right\}+\sum_{j=0}^{r_{1}} \operatorname{Pr}\left\{\left\|\left(0, \delta_{t}\right)\right\| \geq r+1-j,\left\|\left(\delta_{t}, a_{t}\right)\right\|=j\right\}=O\left(\delta_{t}^{2}\right)$, we obtain

$$
=\frac{(r+1) \delta_{t} x}{t} \sum_{j=0}^{\lfloor(r+1) / 2\rfloor} \frac{2^{r+1-2 j} f_{2}(0,0)^{j}}{j !(r+1-2 j) !}(1+o(1)) .
$$

This completes the proof of Lemma 5.

For the analysis of the minimum $r$-scan length, Lemmas 6 and 7 provide the necessary estimates under the continuity assumption of the density $g$. Lemma 6 gives the results relevant to Property 1 , describing the convergence in probability of $n_{t}^{-}\left(a_{t}\right)$ to $N_{t}^{-}\left(a_{t}\right)$. Lemma 7 is required as an error bound of $u_{2}$ in (1) when applying the Chen-Stein method to $n_{t}^{-}\left(a_{t}\right)$.

Proof of Lemma 6. The estimates (12) and (13) give the bounds of events

$$
\begin{aligned}
& \left\{\left\|\left(0, \delta_{t}\right)\right\| \geq 2,\left\|\left(\delta_{t}, a_{t}\right)\right\| \geq r-1\right\} \text { and } \\
& \left\{\left\|\left(0, \delta_{t}\right)\right\|=1,\left\|\left(0, a_{t}\right)\right\|<r+1,\left\|\left(0, a_{t}+\delta_{t}\right)\right\| \geq r+1\right\},
\end{aligned}
$$

respectively. As discussed in Property 1 , their value contributes to the estimate of the probability of the event $\left\{n_{t}^{-}\left(a_{t}\right) \neq N_{t}^{-}\left(a_{t}\right)\right\}$,

$$
\begin{aligned}
\operatorname{Pr}\{\| & \left.\left(0, \delta_{t}\right)\|\geq 2,\|\left(\delta_{t}, a_{t}\right) \| \geq r-1\right\} \\
& \leq \operatorname{Pr}\left\{\left\|\left(0, \delta_{t}\right)\right\| \geq 2\right\} \\
& =\operatorname{Pr}\left\{\left\|A_{1}\left(0, \delta_{t}\right)\right\| \geq 1\right\}+\operatorname{Pr}\left\{\left\|A_{1}\left(0, \delta_{t}\right)\right\|=0,\left\|A_{1}\left(0, \delta_{t}\right)\right\| \geq 2\right\} \\
& =O\left(\delta^{2}\right) .
\end{aligned}
$$


and this proves the bound in (12). Paraphrasing the argument above, we can also prove the bound in (13),

$$
\begin{aligned}
\operatorname{Pr}\{\| & \left.\left(0, \delta_{t}\right)\|=1,\|\left(0, a_{t}\right)\|<r+1,\|\left(0, a_{t}+\delta_{t}\right) \| \geq r+1\right\} \\
& \leq \operatorname{Pr}\left\{\left\|\left(0, \delta_{t}\right)\right\|=1,\left\|\left(\delta_{t}, a_{t}\right)\right\|<r,\left\|\left(a_{t}, a_{t}+\delta_{t}\right)\right\| \geq 1\right\} \\
& <\operatorname{Pr}\left\{\left\|\left(0, \delta_{t}\right)\right\|=1,\left\|\left(a_{t}, a_{t}+\delta_{t}\right)\right\| \geq 1\right\} \\
& =O\left(\delta_{t}^{2}\right) .
\end{aligned}
$$

Proof of Lemma 7. Paraphrasing the argument of (39), for $2 \leq i \leq\left\lfloor a_{t} / \delta_{t}\right\rfloor+1$, we have

$$
\begin{aligned}
\mathrm{E}\left[Z_{1}^{-}\right. & \left.\left(a_{t}\right) Z_{i}^{-}\left(a_{t}\right)\right] \\
& =\operatorname{Pr}\left\{\left\|\left(0, \delta_{t}\right)\right\|=1,\left\|\left(\delta_{t}, a_{t}\right)\right\| \geq r\left\|\left((i-1) \delta_{t}, i \delta_{t}\right)\right\|=1,\left\|\left(i \delta_{t},(i-1) \delta_{t}+a_{t}\right)\right\| \geq r\right\} \\
& \leq \operatorname{Pr}\left\{\left\|\left(0, \delta_{t}\right)\right\|=1,\left\|\left((i-1) \delta_{t}, i \delta_{t}\right)\right\|=1,\left\|\left(i \delta_{t},(i-1) \delta_{t}+a_{t}\right)\right\| \geq r\right\} \\
& =O\left(\delta_{t} \delta_{t} a_{t}^{r}\right) .
\end{aligned}
$$

This proves the bound in (14).

\section{References}

Arratia, R., Goldstein, L. And Gordon, L. (1989). Two moments suffice for Poisson approximations: the Chen-Stein method. Ann. Prob. 17, 9-25.

Barbour, A. D., Holst, L. And Janson, S. (1992). Poisson Approximation. Oxford University Press.

Berg, D. E. ANd Howe, M. M. (1989). Mobile DNA. American Society for Microbiology, Washington, DC.

BERnARDI, G. et al. (1985). The mosaic genome of warm-blooded vertebrates. Science 228, 953-958.

Bernardi, G., Mouchiroud, D., Gautier, C., Bernardi, G. (1988). Compositional patterns in vertebrate genomes: conservation and change in evolution. J. Molec. Evol. 28, 7-18.

BIRD, A. P. (1986). CpG-rich islands and the function of DNA methylation. Nature 321, 209-213.

Blackburn, E. H. (1991). Structure and function of telomeres. Nature 350, 569-573.

Burge, C., Campbell, A. And Karlin, S. (1992). Over- and under-representation of short oligonucleotides in DNA sequences. Proc. Nat. Acad. Sci. USA 89, 1358-1362.

Chen, L. H. Y. (1975). Poisson approximation for dependent trials. Ann Prob. 3, 534-545.

Dembo, A. And Karlin, S. (1992). Poisson approximations for $r$-scan processes. Ann. Appl. Prob. 2, 329-337.

FICKET, J. W. (1982). Recognition of protein coding regions in DNA sequences. Nucleic Acids Res. 10, 5303-5318.

GERSTEIN, M. (1997). A structure census of genomes: comparing bacterial, eukaryotic, and archaeal genomes in terms of protein structure. J. Molec. Biol. 274, 562-576.

GILson, E. et al. (1991). Palindromic units are part of a new bacterial interspersed mosaic element (BIME). Nucleic Acids Res. 19, 1375-1383.

Glaz, J., Naus, J. and Wallenstein, S. (2001). Scan Statistics. Springer, New York.

Josse, J., Kaiser, A. D. And Kornberg, A. (1961). Enzymatic synthesis of deoxyribonucleic acid. J. Biol. Chem. 236, 864-875.

KarLin, S. And Brendel, V. (1992). Chance and statistical significance in protein and DNA sequence analysis. Science 257, 39-49.

Karlin, S. And Cardon, L. R. (1994). Computational DNA sequence analysis. Ann. Rev. Microbiol. 48, $619-654$.

Karlin, S. AND MACKEn, C. (1991). Some statistical problems in the assessment of inhomogeneities of DNA sequence data. J. Amer. Statist. Assoc. 86, 27-35.

Karlin, S., MrázeK, J. and Campbell, A. (1996). Frequent oligonucleotides and peptides of the Haemophilus influenzae genome. Nucleic Acids Res. 24, 4263-4272.

Kingman, J. F. C. (1993). Poisson Processes. Oxford University Press.

Krawiec, S. AND RILEY, M. (1990). Organization of the bacterial chromosome. Microbiol. Rev. 54, 502-539.

NAus, J. I. (1979). An indexed bibliography of clusters, clumps and coincidences. Internat. Statist. Rev. 47, 47-78.

NAUS, J. I. (1982). Approximations for distributions of scan statistics. J. Amer. Statist. Assoc. 77, 177-183.

Reinert, G. and Schbath, S. (1998). Compound Poisson and Poisson approximations for occurrences of multiple words in Markov chains. J. Comput. Biol. 5, 223-254.

WiLlaRd, H. F. AND WAYE, J. S. (1987). Hierachical order in chromosome-specific human alpha satellite DNA. Trends Genet. 3, 192-198. 